NBER WORKING PAPER SERIES

\title{
DIAGNOSTIC EXPECTATIONS AND STOCK RETURNS
}

\author{
Pedro Bordalo \\ Nicola Gennaioli \\ Rafael La Porta \\ Andrei Shleifer \\ Working Paper 23863 \\ http://www.nber.org/papers/w23863 \\ NATIONAL BUREAU OF ECONOMIC RESEARCH \\ 1050 Massachusetts Avenue \\ Cambridge, MA 02138 \\ September 2017
}

Gennaioli thanks the European Research Council and Shleifer thanks the Pershing Square Venture Fund for Research on the Foundations of Human Behavior for financial support of this research. We are grateful to seminar participants at Brown University and Sloan School, and especially to Josh Schwartzstein, Jesse Shapiro, Pietro Veronesi, and Yang You for helpful comments. We also thank V. V. Chari, who encouraged us to confront our model of diagnostic expectations with the Kalman filter. The views expressed herein are those of the authors and do not necessarily reflect the views of the National Bureau of Economic Research.

NBER working papers are circulated for discussion and comment purposes. They have not been peer-reviewed or been subject to the review by the NBER Board of Directors that accompanies official NBER publications.

(C) 2017 by Pedro Bordalo, Nicola Gennaioli, Rafael La Porta, and Andrei Shleifer. All rights reserved. Short sections of text, not to exceed two paragraphs, may be quoted without explicit permission provided that full credit, including $(\odot$ notice, is given to the source. 
Diagnostic Expectations and Stock Returns

Pedro Bordalo, Nicola Gennaioli, Rafael La Porta, and Andrei Shleifer

NBER Working Paper No. 23863

September 2017

JEL No. D03,D84,G02,G12

\begin{abstract}
We revisit La Porta's (1996) finding that returns on stocks with the most optimistic analyst long term earnings growth forecasts are substantially lower than those for stocks with the most pessimistic forecasts. We document that this finding still holds, and present several further facts about the joint dynamics of fundamentals, expectations, and returns for these portfolios. We explain these facts using a new model of belief formation based on a portable formalization of the representativeness heuristic. In this model, analysts forecast future fundamentals from the history of earnings growth, but they over-react to news by exaggerating the probability of states that have become objectively more likely. Intuitively, fast earnings growth predicts future Googles but not as many as analysts believe. We test predictions that distinguish this mechanism from both Bayesian learning and adaptive expectations, and find supportive evidence. A calibration of the model offers a satisfactory account of the key patterns in fundamentals, expectations, and returns.
\end{abstract}

Pedro Bordalo

Saïd Business School

University of Oxford

Park End Street

Oxford, OX1 1HP

United Kingdom

pedro.bordalo@sbs.ox.ac.uk

Nicola Gennaioli

Department of Finance

Università Bocconi

Via Roentgen 1

20136 Milan, Italy

nicola.gennaioli@unibocconi.it

\author{
Rafael La Porta \\ Brown University \\ 70 Waterman Street \\ Room 101 \\ Providence, RI 02912 \\ and NBER \\ rafael.laporta@brown.edu \\ Andrei Shleifer \\ Department of Economics \\ Harvard University \\ Littauer Center M-9 \\ Cambridge, MA 02138 \\ and NBER \\ ashleifer@harvard.edu
}




\section{Introduction}

La Porta (1996) shows that expectations of stock market analysts about long-term earnings growth of the companies they cover have strong predictive power for these companies' future stock returns. Companies whose earnings growth analysts are most optimistic about earn poor returns relative to companies whose earnings growth analysts are most pessimistic about.

Figure 1 offers an update of this phenomenon. Stocks are sorted by analyst long-term earnings per share growth forecasts (LTG). The LLTG portfolio is the 10\% of stocks with most pessimistic forecasts, the HLTG portfolio is the $10 \%$ of stocks with most optimistic forecasts. The figure reports geometric averages of one-year returns on equally weighted portfolios.

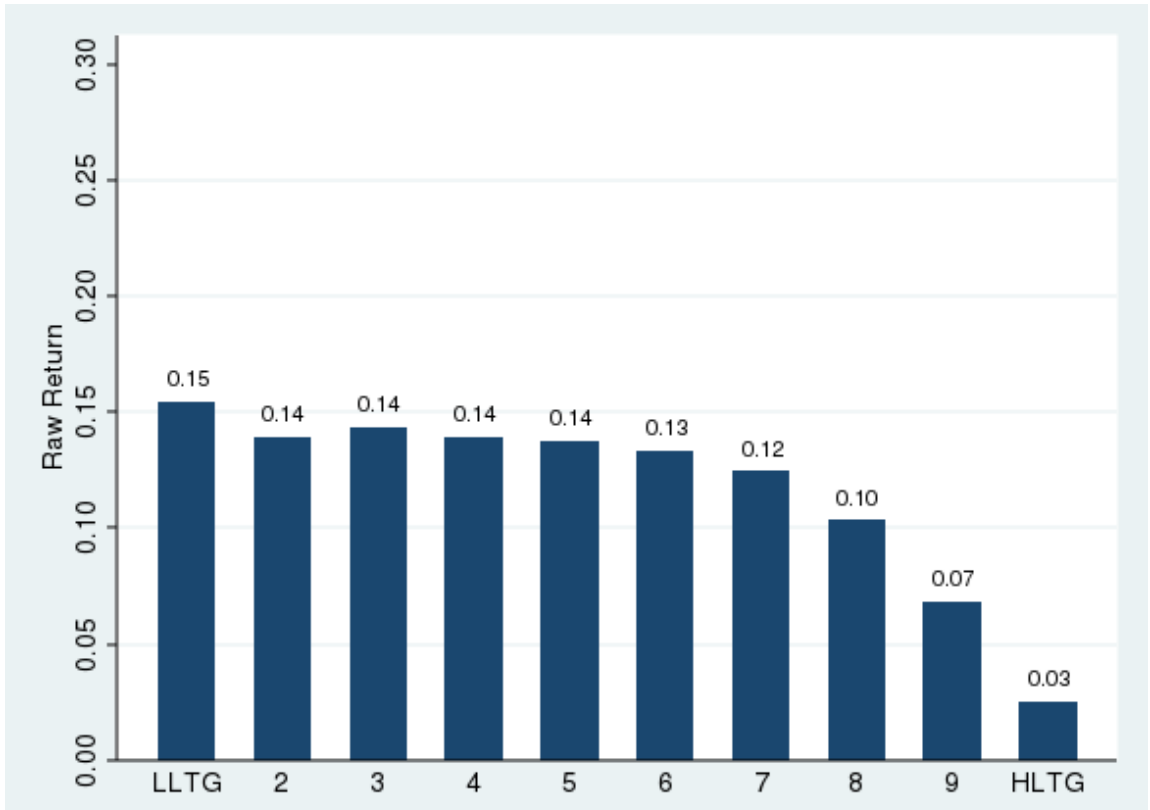

Figure 1. Annual Returns for Portfolios Formed on LTG. In December of each year between 1981 and 2015, we form decile portfolios based on ranked analysts' expected growth in earnings per share and report the geometric average one-year return over the subsequent calendar year for equally-weighted portfolios with monthly rebalancing.

Consistent with La Porta (1996), the LLTG portfolio earns an average return of $15 \%$ in the year after formation, while the HLTG portfolio earns only $3 \% .^{2}$ Adjusting for systematic risk

\footnotetext{
${ }^{2}$ The spread in Figure 1 is in line with, although smaller than, previous findings. LaPorta (1996) finds an average yearly spread of $20 \%$ but employed a shorter sample (1982 to 1991). Dechow and Sloan (1997) use a similar sample to La Porta (1996) and find a 15\% spread. Appendix A shows that the spread also holds in sample subperiods.
} 
deepens the puzzle: the HLTG portfolio has higher market beta than the LLTG portfolio, and performs much worse in market downturns. ${ }^{3}$ Over the past 35 years, betting against extreme analyst optimism has been on average a good idea. La Porta (1996) interprets this finding as evidence that analysts, as well as investors who follow them or think like them, are too optimistic about stocks with rapidly growing earnings, and too pessimistic about stocks with deteriorating earnings.

In this paper we analyze the dynamics of expectation formation and offer a psychologically founded theory that jointly accounts for the behavior of fundamentals, expectations, and returns. We propose a new learning model in which beliefs are forward looking just as with rational expectations, but distorted by representativeness, which biases the interpretation of the news. Specifically, analysts update excessively in the direction of states of the world whose objective likelihood rises the most in light of the news. The model delivers overreaction to news and extrapolation. It also makes sharp predictions that distinguish it from both Bayesian learning and mechanical adaptive expectations. We test, and confirm, several of these new predictions.

After describing the data in Section II, in Section III we document three facts. First, HLTG stocks exhibit fast past earnings growth, which slows down going forward. Second, forecasts of future earnings growth of HLTG stocks are excessively optimistic, and are systematically revised downward later. Third, HLTG stocks exhibit good past returns but their average returns going forward are low. The opposite dynamics obtain for LLTG stocks, but in a much less extreme form, an asymmetry we do not account for in our model.

\footnotetext{
${ }^{3} \mathrm{We}$ find $\beta_{H L T G}=1.51$, and $\beta_{L L T G}=0.78$ (Appendix A). The HLTG-LLTG spread holds within size buckets and it is strongest for intermediate $\mathrm{B} / \mathrm{M}$ levels.
} 
Our model of learning in Section IV is based on Gennaioli and Shleifer's (GS, 2010) formalization of Kahneman and Tversky's (1972) representativeness heuristic. As in GS (2010) and Bordalo, Coffman, Gennaioli and Shleifer (BCGS, 2016), a trait $t$ is representative of a group $G$ when it occurs more frequently in that group than in a reference group $-G$. The representative trait $t$ is quickly recalled and its frequency in group $G$ is exaggerated. To illustrate, consider a doctor assessing the health status of a patient after a positive test. The representative patient is $t=$ "sick", because sick people are more frequent among patients who tested positive than in the overall population. The sick patient type quickly comes to mind and the doctor inflates its probability, which in reality may be low if the disease is rare.

In the present setting, analysts learn about firms' unobserved fundamentals on the basis of a noisy signal (e.g., current earnings). The rational benchmark is the Kalman Filter. Relative to this benchmark, representativeness causes analysts to inflate the probability of firm types whose likelihood has increased the most in light of recent earning news. After exceptionally high earnings growth, the representative firm is a "Google", and analysts inflate its probability. There is a kernel of truth: Googles are truly more likely among firms exhibiting exceptional growth. Beliefs, however, go too far: Googles are quite rare in absolute terms. Following our work on credit cycles (BGS 2017), we say that this distorted inference follows a "Diagnostic Kalman Filter" to emphasize that it overweighs information diagnostic of certain firm types.

Section V maps the model to the data. It starts by considering a key implication of the kernel of truth hypothesis: expectations exaggerate the incidence of Googles in the HLTG group because these firms are relatively more likely there. The data confirms that the HLTG group has a fatter right tail of strong future performers than all other firms. These exceptional performers are thus representative of the HLTG group, even though they are unlikely in absolute terms. As the 
model predicts, we also show that analysts vastly exaggerate the share of firms with exceptional earnings growth in the HLTG group. We then show that the model qualitatively accounts for the joint dynamics of fundamentals, expectations, and returns documented in Section III. Intuitively, because strong eps growth is diagnostic of future strong growth, analysts become excessively optimistic about HLTG firms, driving up prices and generating negative forecast errors. Returns are low post formation as analysts correct their inflated forecasts.

Section VI performs three additional exercises. Section VI.A shows that the inflated expectations about HLTG stocks are due to over-reaction to good news. In particular, we find that upward revisions in LTG forecasts are associated with excessive optimism (Coibion and Gorodnichenko 2015). Section VI.B calibrates model parameters using data on the autocorrelation of earnings and the over-reaction estimates of Section VI.A. Our simple model does a good job at quantitatively accounting for the link between forecast errors and abnormal returns documented in Section III. Section VI.C shows that the dynamics of expectations are hard to explain using mechanical extrapolation: expectations mean revert even without news, suggesting that analysts are forward looking in incorporating fundamental mean reversion into their forecasts.

Our paper is related to several strands of research in finance. Empirical research on crosssectional stock return predictability is framed in terms of concepts such as extrapolation (e.g., DeBondt and Thaler 1985, 1987, Cutler, Poterba and Summers 1991, Lakonishok, Shleifer, and Vishny 1994, Dechow and Sloan 1997), but most studies in this area do not use expectations data. Some older studies in finance that do use expectations data include Dominguez (1986) and Frankel and Froot $(1987,1988)$. A large literature on analyst expectations shows that they are on average too optimistic (Easterwood and Nutt 1999, Michaely and Womack 1999, Dechow, Hutton, and Sloan 2000). More recently, the use of survey expectations data not just by analysts 
but also by investors has been making a comeback (e.g., Ben David, Graham and Harvey 2013, Greenwood and Shleifer 2014, Gennaioli, Ma, and Shleifer 2015).

Bouchaud, Landier, Krueger and Thesmar (2016) use analyst expectations data to study the profitability anomaly, and offer a model in which expectations under-react to news, in contrast with our focus on over-reaction. As we show in Section VI.A, in our data there is also some shortterm under-reaction, but at the long horizons of LTG forecasts over-reaction prevails. Daniel, Klos, and Rottke (2017) show that stocks featuring high dispersion in analyst expectations and high illiquidity earn high returns, but do not offer a theory of expectations and their dispersion.

Our paper is also related to research on over-reaction and volatility, which begins with Shiller (1981), DeBondt and Thaler (1985, 1987), Cutler, Poterba, and Summers (1990), and DeLong et al. (1990a). This work assumes mechanical, backward looking rules for belief updating, based either on adaptive expectations (e.g., DeLong et al 1990b, Barsky and DeLong 1993, Barberis and Shleifer 2003, Barberis et al. 2015, Glaeser and Nathanson 2015), adaptive learning (Marcet and Sargent 1989, Adam, Marcet, and Nicolini 2016, Adam, Marcet, and Beutel 2017), or rules of thumb (Hong and Stein, 1999). ${ }^{4}$ Pastor and Veronesi $(2003,2005)$ present rational learning models in which uncertainty about the fundamentals of some firms boosts the volatility of their returns and their market to book ratios. Under some conditions, learning dynamics also explain predictability in aggregate stock returns (Pastor and Veronesi 2006). This approach does not analyze expectations data or cross sectional differences in returns. Barberis, Shleifer, and Vishny (BSV, 1998), Daniel, Hirshleifer, and Subramanyam (DHS, 1998) and Odean (1998) offer models grounded in psychology. The BSV model is motivated by representativeness, and we return to it in Section VI.C. DHS (1998) and Odean (1998) build a

\footnotetext{
${ }^{4}$ In Adam, Marcet, and Beutel (2017), agents learn about the mapping between fundamentals and price outcomes, but hold rational expectations of fundamentals. While this approach is complementary to ours, it does not address the evidence on expectations of fundamentals that is central to our paper.
} 
model of investor overconfidence, the tendency of decision-makers to exaggerate the precision of private information, which causes divergence in beliefs and excess trading. In contrast, our model is concerned with overreaction to public information.

One advantage of our approach worth stressing is that our model is not designed for a specific finance setting but is more portable (Rabin 2013). Our formalization of representativeness was developed to account for biases in general probability assessments such as base rate neglect, conjunction and disjunction fallacies in a laboratory context (Gennaioli and Shleifer 2010). We have previously applied it to modeling social stereotypes (Bordalo, Coffman, Gennaioli and Shleifer 2016, 2017) and credit cycles (Bordalo, Gennaioli and Shleifer 2017), where the patterns of over-reaction to news, systematic forecast errors, expectations revisions, and predictable returns are similar to those discussed here.

\section{Data and Summary Statistics}

\section{II.A. Data}

We gather data on analysts' expectations from IBES, stock prices and returns from CRSP, and accounting information from CRSP/COMPUSTAT. Below we describe the measures used in the paper and, in parentheses, provide their mnemonics in the primary datasets.

From the IBES Unadjusted US Summary Statistics file we obtain mean analysts' forecasts for earnings per share and their expected long-run growth rate (meanest, henceforth "LTG") for the period December 1981, when LTG becomes available, through December 2016. IBES defines LTG as the "expected annual increase in operating earnings over the company's next full business cycle", a period ranging from three to five years. From the IBES Detail History Tape file we get analyst-level data on earnings forecasts. We use CRSP daily data on stock splits (cfacshr) to 
adjust IBES earnings per share figures. On December of each year between 1981 and 2015, we form LTG decile portfolios based on stocks that report earnings in US dollars. ${ }^{5}$

The CRSP sample includes all domestic common stocks listed on a major US stock exchange (i.e. NYSE, AMEX, and NASDAQ) except for closed-end funds and REITs. Our sample starts in 1978 and ends in 2016. We present results for both buy-and-hold annual returns and daily cumulative-abnormal returns for various earnings' announcement windows. We compute annual stock returns by compounding monthly returns. We focus on equally-weighted returns for LTG portfolios. If a stock is delisted, CRSP tries to establish its price after delisting. Whenever a post-delisting price exists, we use it in the computations for returns. When CRSP is unable to determine the value of a stock after delisting, we assume that the investor was able to trade at the last quoted price. After a stock disappears from the sample, we replace its return until the end of the calendar year with the return of the equally-weighted market portfolio. Given that IBES surveys analysts around the middle of the month (on Thursday of the third week of the month), LTG is in the information set when we form portfolios. Daily cumulative abnormal returns are defined relative to CRSP's equally-weighted index. We also gather data on market capitalization in December of year $t$ as well as the pre-formation 3-year return ending on December of year $t$. Finally, we rank stocks into deciles based on market capitalization using breakpoints for NYSE stocks.

We get from the CRSP/COMPUSTAT merged file on assets (at), sales (sale), net income (ni), book equity, common shares used to calculate earnings per share (cshpri), adjustment factor for stock splits (adjex_f), and Wall Street Journal dates for quarterly earnings' releases (rdq). Our CRSP/COMPUSTAT data covers the period 1978-2016. We use annual and quarterly accounting

\footnotetext{
${ }^{5}$ We form portfolios in December of each year, because that is when IBES data on analyst expectations is released. Unlike in Fama and French (1993) we know exactly when the information required for an investable strategy is public.
} 
data. We define book equity as stockholders' equity (depending on data availability seq, ceq+pfd, or $a t-l t$ ) plus deferred taxes (depending on data availability $t x d i t c$ or $t x d b+i t c b$ ) minus preferred equity (depending on data availability $p s t k r, p s t k l$, or $p s t k$ ). We define operating margin as the difference between sales and cost of goods sold $(\operatorname{cog} s)$ and return on equity as net income divided by book equity. We compute the annual growth rate in sales per share in the most recent 3 fiscal years. When merging IBES with CRSP/COMPUSTAT, we follow the literature and assume that data for fiscal periods ending after June becomes available during the next calendar year.

\section{II.B. Summary Statistics}

Table 1 reports the means of some of the variables for LTG decile portfolios. The number of stocks with CRSP data on stock returns and IBES data on LTG varies by year, ranging from 1,310 in 1981 to 3,849 in 1997 . On average, each LTG portfolio contains 241 stocks. The forecasted growth rate in earnings per share ranges from $4 \%$ for the lowest LTG decile (LLTG) to 38\% for the highest decile (HLTG), an enormous difference. LLTG stocks are larger than HLTG stocks in terms of both total assets (7,942 MM vs. 1,081 MM) and market capitalization $(3,913$ MM vs. 1,749 MM). However, differences in size are not extreme: the average size decile is 5.1 for LLTG and 3.6 for HLTG.

LLTG stocks have lower operating margins to asset ratios than HLTG stocks but higher return on equity (5\% vs $-6 \%$ ). In fact, $31 \%$ of HLTG firms have negative eps while the same is true for only $12 \%$ of LLTG stocks. The high incidence of negative eps companies in the HLTG portfolio underscores the importance of the definition of LTG in terms of annual earnings growth over a full business cycle. Current negative earnings do not hinder these firms' future prospects. 


\section{Table 1 - Descriptive Statistics for Portfolios Formed on $L T G$.}

We form decile portfolios based on analysts' expected growth in earnings per share (LTG) in December of each year between 1981 and 2015. The table reports time-series means of the variables described below for equally-weighted $L T G$ portfolios. Unless otherwise noted, accounting variables pertain to the most recently available fiscal year, where we follow the standard assumption that data for fiscal periods ending after June become available during the next calendar year. Assets is book value of total assets (in millions). Market capitalization is the value of common stock on the last trading day of year $t$ (in millions). Size decile refers to deciles of market capitalization with breakpoints computed using only NYSE stocks. Operating margin to assets is the difference between sales and cost of goods sold divided by assets. Return on equity is net income divided by book equity. Percent eps positive is the fraction of firms with positive earnings. Observations is the number of observations in a year. All variables are capped at the $1 \%$ and $99 \%$ levels.

LTG decile

\begin{tabular}{lcccccccccc}
\hline & 1 & 2 & 3 & 4 & 5 & 6 & 7 & 8 & 9 & 10 \\
\hline Expected growth in eps (LTG) & $4 \%$ & $9 \%$ & $10 \%$ & $12 \%$ & $14 \%$ & $15 \%$ & $17 \%$ & $20 \%$ & $25 \%$ & $38 \%$ \\
Assets (MM) & 7,942 & 10,570 & 10,391 & 7,631 & 5,395 & 3,591 & 2,352 & 1,903 & 1,295 & 1,084 \\
Market capitalization (MM) & 3,913 & 4,829 & 5,434 & 4,552 & 4,218 & 3,406 & 2,685 & 2,596 & 1,818 & 1,749 \\
Size decile & 5.1 & 5.1 & 5.4 & 5.3 & 5.2 & 4.7 & 4.5 & 4.0 & 3.7 & 3.6 \\
Operating margin to assets & $19 \%$ & $25 \%$ & $29 \%$ & $33 \%$ & $37 \%$ & $40 \%$ & $42 \%$ & $42 \%$ & $41 \%$ & $36 \%$ \\
Return on equity & $5 \%$ & $8 \%$ & $11 \%$ & $10 \%$ & $10 \%$ & $9 \%$ & $9 \%$ & $6 \%$ & $1 \%$ & $-6 \%$ \\
Percent eps positive & $88 \%$ & $90 \%$ & $93 \%$ & $93 \%$ & $93 \%$ & $91 \%$ & $91 \%$ & $87 \%$ & $81 \%$ & $69 \%$ \\
Observations & 257 & 245 & 235 & 242 & 243 & 257 & 216 & 253 & 240 & 227 \\
\hline
\end{tabular}

\section{A New Look at the Data.}

Figure 1 suggests that analysts and the stock market may be too bullish on firms they are optimistic about, and too bearish on firms they are pessimistic about. To assess this possibility, we document some basic facts connecting firms' performance, expectations and returns.

Figure 2 reports average earnings per share of HLTG and LLTG portfolios in years $t-3$ to $t+3$ where $t=0$ corresponds to portfolio formation. We normalize year $t-3$ earnings per share of both portfolios to $\$ 1$. Earnings per share for HLTG stocks exhibit explosive growth during the pre-formation period, rising from $\$ 1$ in year -3 to $\$ 1.56$ in year 0. Earnings of LLTG firms decline to $\$ 0.87$ during the corresponding period. But the past does not repeat itself after portfolio formation, because of mean reversion in earnings growth. Earnings growth of HLTG 
firms slows down, while earnings of LLTG firms recover during the post-formation period. HLTG firms remain more profitable on average than LLTG firms 3 years after portfolio formation, but the difference in actual growth rates is nowhere near the difference in LTG exhibited in Table 1.

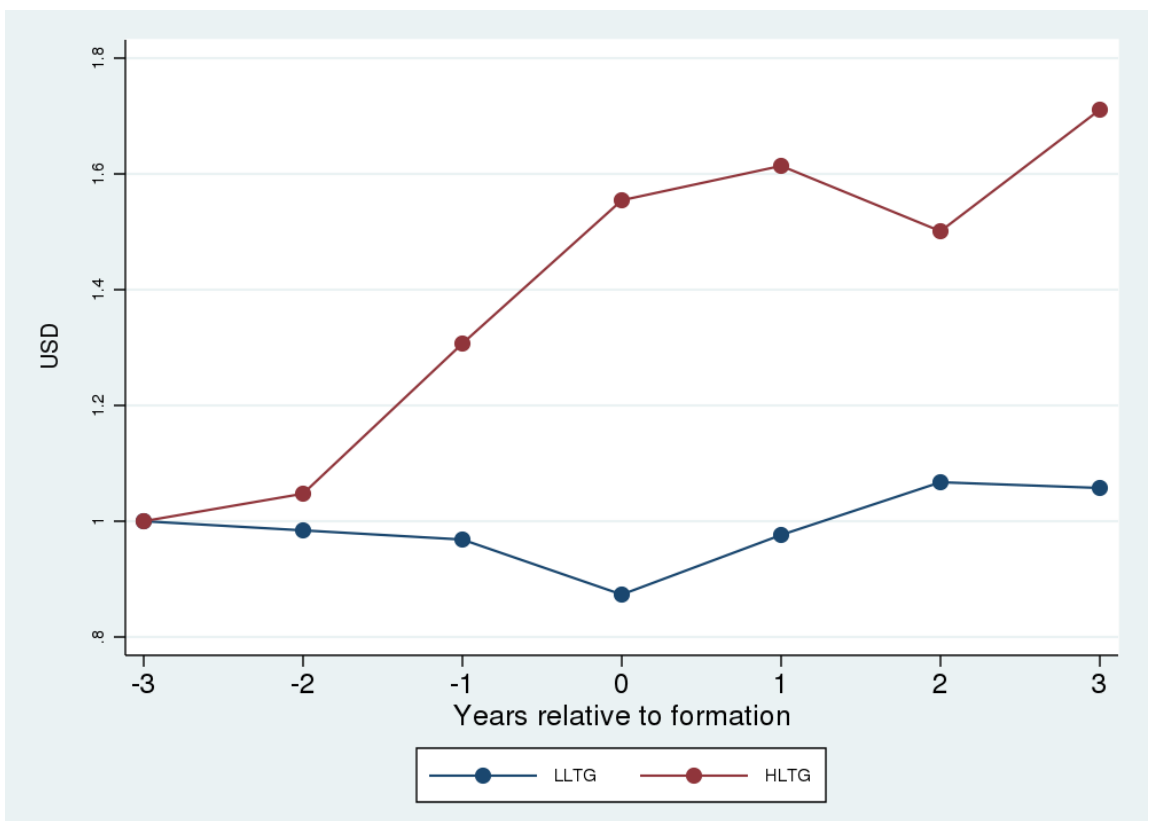

Figure 2. Evolution of EPS. In December of years $(t)$ 1981, 1984., ..,2011, and 2013, we form decile portfolios based on ranked analysts' expected growth in earnings per share (LTG). We report the mean value of earnings per share for the highest (HLTG) and lowest (LLTG) LTG deciles for each year between $t-3$ and $t+3$. We exclude firms with negative earnings in $\mathrm{t}-3$ and normalize to 1 the value of earnings per share in $\mathrm{t}-3$.

Figure 3 shows the average LTG for the HLTG and LLTG portfolios over the same time window. Prior to portfolio formation, expectations of long-term growth for HLTG firms rise dramatically in response to strong earnings growth (compare with Figure 2), while expectations for LLTG drop. After formation, expectations for HLTG firms are revised sharply downwards, particularly during the first year, whereas LTG of LLTG firms is revised moderately up. Three years after portfolio formation, earnings of HLTG firms are still expected to grow faster than those of LLTG firms, but the spread in expected growth rates of earnings has narrowed considerably. One potential concern about Figure 2 and in other Figures in this Section is the role of attrition in the sample. Reassuringly, the level of attrition is similar across HLTG and LLTG 
portfolios (roughly $30 \%$ three years post formation), and the key features of the Figures continue to hold when we restrict the sample to firms that survive for five years post formation.

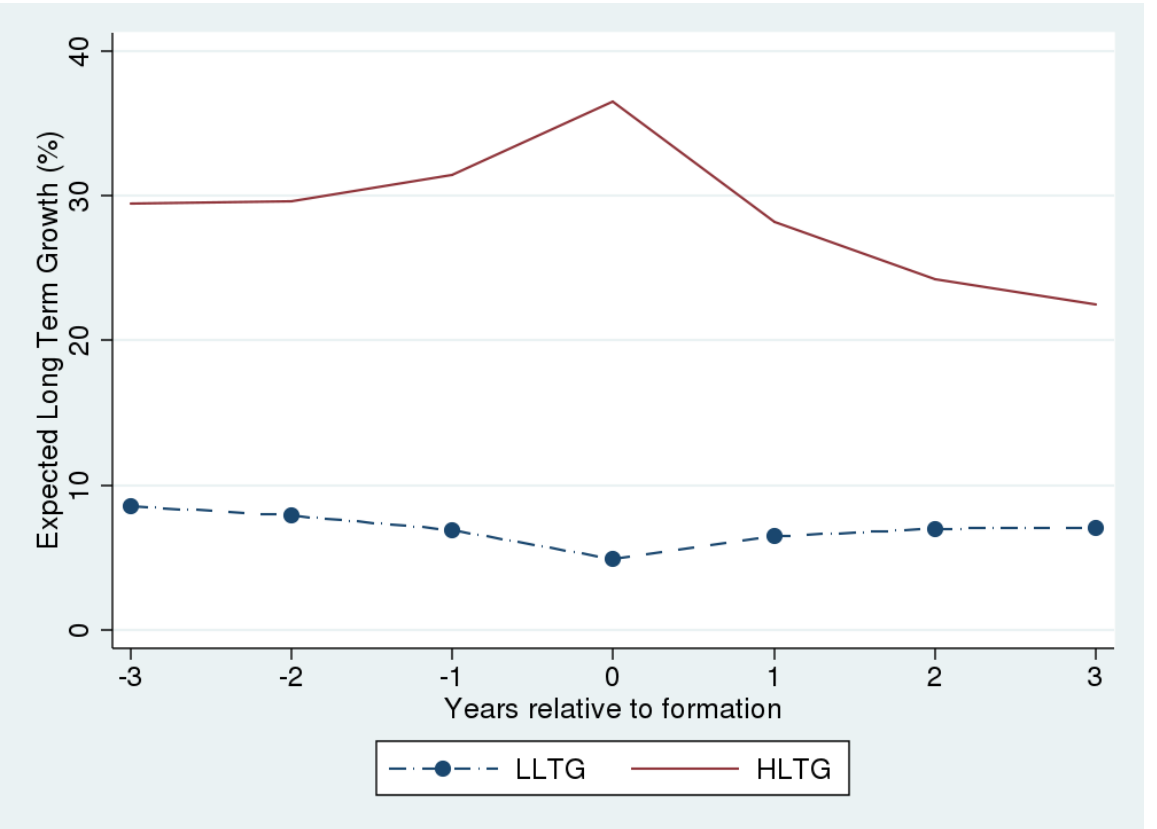

Figure 3. Evolution of LTG. In December of each year $t$ between 1984 and 2013, we form decile portfolios based on ranked analysts' expected growth in earnings per share (LTG) and report the mean value of LTG on December of years $t-3$ to $t+3$ for the highest (HLTG) and lowest (LLTG) LTG deciles. We include in the sample stocks with LTG forecasts in year $t-3$. Values for $t+1, t+2$, and $t+3$ are based on stocks with IBES coverage for those periods.

Expectations of long-term growth follow the pattern of actual earnings per share of HLTG and LLTG portfolios displayed in Figure 2. Analysts seem to be learning about firms' earnings growth from their past performance. Fast pre-formation growth leads analysts to place a firm in the HLTG category. Post-formation growth slowdown triggers a downward revision of forecasts.

Mean reversion in forecasts may be caused by mean reversion in fundamentals, which is evident in Figure 2, or by the correction of analysts' expectations errors at formation. To see the role of expectations errors, Figure 4 reports the difference between realized earnings growth and analysts' LTG expectations in each portfolio, from formation to year $t+3$. There is strong overoptimism, i.e. very negative forecast errors, for HLTG firms. There is also over-optimism for 
LLTG firms, consistent with many previous studies and usually explained by distorted analyst incentives (e.g., Dechow et al. 2000, Easterwood and Nutt 1999, Michaely and Womack 1999).

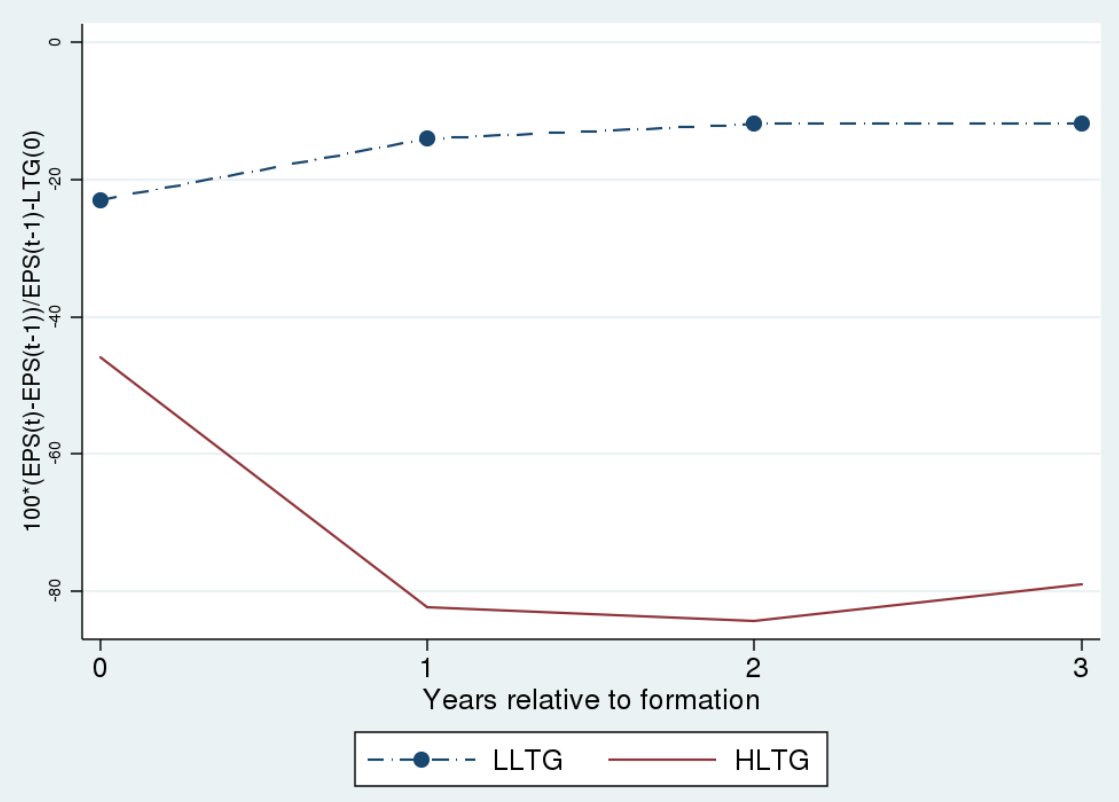

Figure 4. Realized earnings per share vs. Expected growth in long-term earnings. In December of each year $t$ between 1981 and 2013 we form decile portfolios based on ranked analysts' expected growth in eps. We plot the difference between the annual growth rate in earnings per share in each year between $t$ and $\mathrm{t}+3$ and the forecast for long-term growth in earnings made in year $t$.

The overestimation of earnings growth for HLTG firms is economically large. By year 3, actual earnings are a small fraction of what analysts forecast: earnings per share grow from 0.16 upon formation to 0.21 in $t+3$, compared to the prediction of 0.70 based on LTG at formation.

There are two concerns with this evidence, both of which deal with the interpretation and reliability of our expectations data. The first is whether Figure 4 reflects genuine errors in analyst beliefs or alternatively their distorted incentives. For instance, analysts may blindly follow the market, reporting high LTG for firms that investors are excited about. This possibility does not undermine our analysis. To the extent that analysts' forecasts reflect investor beliefs, they are informative about the expectations shaping market prices. The second, and more radical, concern 
is whether investors share analysts' beliefs or alternatively the latter are noise. To address this concern we look at the dynamics of stock returns pre and post formation. Figure 5 shows stock returns around earnings announcements. For every stock in the HLTG and LLTG portfolios, we compute the 12-day cumulative return during the four quarterly earnings announcement days, in years $t-3$ to $t+4$, following the methodology of La Porta et al. (1997).

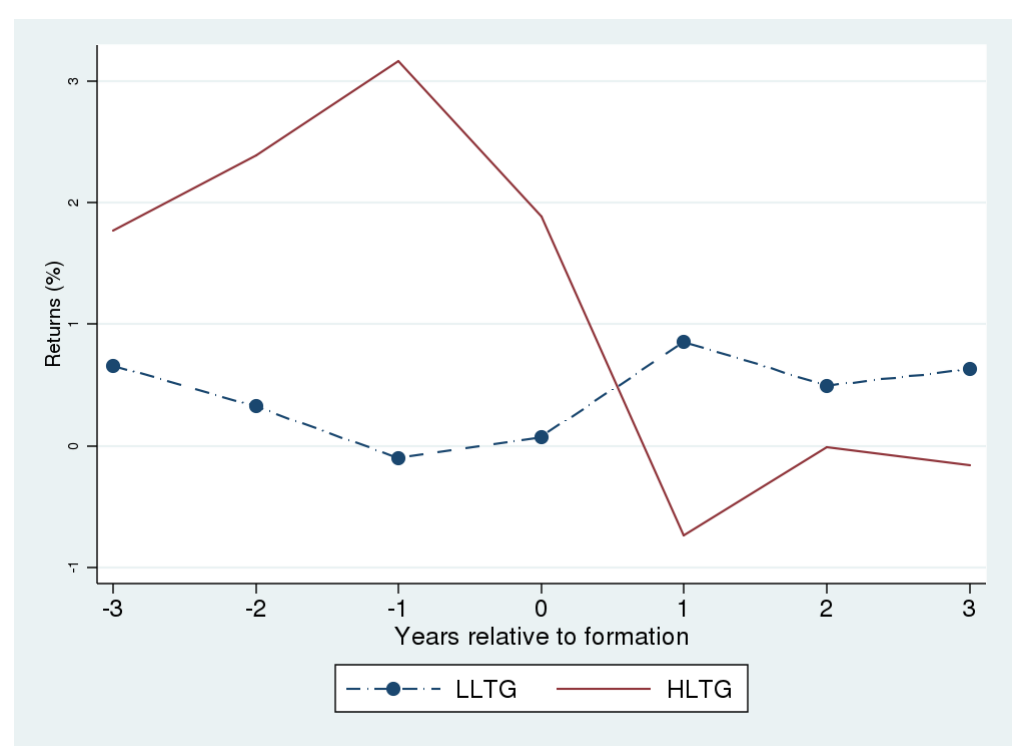

Figure 5. Twelve-day Returns on Earnings Announcements for LTG Portfolios. In December of each year $t$ between 1981 and 2013, we form decile portfolios based on ranked analysts' expected growth in earnings per share. Next, for each stock, we compute the 3-day market-adjusted return centered on earnings announcements in years $t-3, \ldots, t+3$. Next, we compute the annual return that accrues over earnings announcements by compounding all 3-day stock returns in each year. We report the equally-weighted average annual return during earnings announcements for the highest (HLTG) and lowest (LLTG) LTG deciles. Excess returns are defined relative to the equally-weighted CRSP market portfolio.

HLTG stocks positively surprise investors with their earnings announcements in the years prior to portfolio formation, when there is upward revision of LTG. ${ }^{6}$ Returns are low afterwards, especially in year 1, consistent with the sharp decline in LTG in this period. Analysts' overoptimism thus seems to be shared by investors, so that HLTG stocks consistently disappoint in the

\footnotetext{
${ }^{6}$ The persistence of positive surprises and high returns exhibited by the HLTG portfolio in Figure 5 should not be confused with time-series momentum. Instead, it arises from selection: on average, firms require a sequence of positive shocks to be classified as HLTG. The basic HLTG-LLTG spread is orthogonal to short-term return momentum in the 6 months prior to portfolio formation, see Appendix A.
} 
post formation period. The converse holds for LLTG stocks, but in a milder form. In sum, analysts' expectations are not noise: they correlate both with actual earnings and stock returns.

Overall, three aspects of the analyst expectations evidence play a key role in our theory. First, analysts react to news. Optimism about HLTG firms follows the observation of fast earnings growth, and is reversed after earnings' growth slows down. It seems that analysts are trying to learn the earnings generating capacity of firms on the basis of past performance. Second, one should be skeptical of analyst rationality, as shown by the evidence on systematic forecast errors in the HLTG group (which comprises more than 200 firms). Third, the dynamics of returns match the dynamics of expectations and their errors, both pre and post formation.

In the next section, we develop a model based on psychological first principles that sheds light on this evidence. This model takes the perspective that the consensus analyst forecasts are the relevant expectations that shape prices and whose dynamics drive returns. A key goal of our approach is to simultaneously explain analysts' forecast errors and return predictability. Predictable returns as such could arise with full rationality: if investors have different required returns for different firms and if analysts jointly learn a firm's required return and its earnings. ${ }^{7}$ However, the evidence on predictable errors in expectations of both earnings and returns is inconsistent with this possibility. We return to this point in Section VI.

\section{A model of learning with representativeness}

\section{IV.A. The Setup}

\footnotetext{
${ }^{7}$ We thank our discussant Pietro Veronesi for making this point.
} 
There is a measure 1 of firms, $i \in[0,1]$. At time $t$, the natural logarithm of a firm's earnings per share (eps) $x_{i, t}$ is given by:

$$
x_{i, t}=b x_{i, t-1}+f_{i, t}+\epsilon_{i, t}
$$

where $b \in[0,1]$ captures mean-reversion in eps, and $\varepsilon_{i, t}$ denotes a transitory i.i.d. normally distributed shock to eps, $\varepsilon_{i, t} \sim \mathcal{N}\left(0, \sigma_{\varepsilon}^{2}\right)$. The term $f_{i, t}$, which we call the firm's "fundamental", captures the firm's persistent earnings capacity. It obeys the law of motion:

$$
f_{i, t}=a \cdot f_{i, t-1}+\eta_{i, t}
$$

where $a \in[0,1]$ is persistence and $\eta_{i, t} \sim \mathcal{N}\left(0, \sigma_{\eta}^{2}\right)$ is an i.i.d. normally distributed shock that may for instance result from a corporate reorganization or a change in market competition. We can think of firms with exceptionally high $f_{i, t}$ as "Googles" that will produce very high earnings in the future, and firms with low $f_{i, t}$ as "lemons" that will produce low earnings in the future. We assume stationarity of earnings by imposing the additional condition $b \leq a$.

The analyst observes eps $x_{i, t}$ but not the fundamental $f_{i, t}$. The Kalman filter characterizes the forecasted distribution of $f_{i, t}$ at any time $t$ conditional on the firm's past and current earnings $\left(x_{i, u}\right)_{u \leq t}$. Given the mean forecasted fundamental $\hat{f}_{i, t-1}$ for firm $i$ at $t-1$ and its current earnings $x_{i, t}$, the firm's current forecasted fundamental is normally distributed with variance $\sigma_{f}^{2}$ and mean: ${ }^{8}$

$$
\hat{f}_{i, t}=a \hat{f}_{i, t-1}+K\left(x_{i, t}-b x_{i, t-1}-a \hat{f}_{i, t-1}\right),
$$

where $K \equiv \frac{a^{2} \sigma_{f}^{2}+\sigma_{\eta}^{2}}{a^{2} \sigma_{f}^{2}+\sigma_{\eta}^{2}+\sigma_{\varepsilon}^{2}}$ is the signal to noise ratio.

\footnotetext{
${ }^{8}$ Equation (3) arises in the long run, when the variance of fundamentals has converged to its steady state $\sigma_{f}^{2}$. Given the presence of fundamental shocks $\eta_{i, t}$, a firm's fundamental is never learned with certainty. In the long run, variance stays constant at $\sigma_{f}^{2}$, which is defined as the solution to:

$$
a^{2} \sigma_{f}^{4}+\sigma_{f}^{2}\left[\sigma_{\eta}^{2}+\left(1-a^{2}\right) \sigma_{\varepsilon}^{2}\right]-\sigma_{\eta}^{2} \sigma_{\varepsilon}^{2}=0
$$
}


The new forecast of fundamentals starts from the history-based value $a \hat{f}_{i, t-1}$ but adjusts it in the direction of the current surprise $x_{i, t}-b x_{i, t-1}-a \hat{f}_{i, t-1}$. The extent of adjustment increases in $K$. Absent transitory shocks $\left(\sigma_{\varepsilon}^{2}=0\right)$, earnings are perfectly informative about fundamentals and the adjustment is full (i.e., $\hat{f}_{i, t}=x_{i, t}-b x_{i, t-1}$ ). As the importance of transitory shocks rises, earnings become a noisier signal and estimated fundamentals change less with earnings, so $K<1$.

The signal to noise ratio solves the key inference problem here: to separate the extent to which current earnings $x_{i, t}$ are due to persistent or transitory shocks (i.e., $\eta_{i, t}$ versus $\varepsilon_{i, t}$ ). Among episodes of exceptionally high growth, the analyst must try to tell apart those due to luck and those due to the fact that the firm is the next Google.

Equation (3) yields not only the true conditional distribution of fundamentals, but also the assessment of fundamentals performed by a Bayesian agent seeking to forecast future earnings. We next describe how the representativeness heuristic distorts this learning process.

\section{IV.B. Representativeness and the Diagnostic Kalman filter}

Kahneman and Tversky (KT 1972) argue that the automatic use of the representativeness heuristic causes individuals to estimate a type as likely in a group when it is merely representative of that group. KT define representativeness as follows: "an attribute is representative of a class if it is very diagnostic; that is, the relative frequency of this attribute is much higher in that class than in a relevant reference class (TK 1983)." Starting with KT (1972), experimental evidence has found ample support for the role of representativeness.

Gennaioli and Shleifer (2010) propose a model of this phenomenon in which a decision maker assesses the distribution $h(T=\tau \mid G)$ of a variable $T$ in a group $G$. The representativeness of the specific type $\tau$ for $G$ is: 


$$
R(\tau, G) \equiv \frac{h(T=\tau \mid G)}{h(T=\tau \mid-G)}
$$

As in $\mathrm{KT}$, a type is more representative if it is relatively more frequent in $G$ than in the comparison group $-G$. The probability of representative types is overestimated relative to the truth. BCGS (2016) offer a convenient formalization of this process by assuming that probability judgments are formed using the distorted density:

$$
h^{\theta}(T=\tau \mid G)=h(T=\tau \mid G)\left[\frac{h(T=\tau \mid G)}{h(T=\tau \mid-G)}\right]^{\theta} Z,
$$

where $\theta \geq 0$ and $Z$ is a constant ensuring that the distorted density $h^{\theta}(T=\tau \mid G)$ integrates to 1 . The extent of probability distortions increases in $\theta$, with $\theta=0$ capturing the rational benchmark.

In Kahneman and Tversky's quote, as well as in Equation (4), the representativeness of a type depends on its true relative frequency in the group $G$. The distorted probability in (5) thus depends on the true probability, which renders the model empirically testable. GS (2010) interpret this feature on the basis of limited and selective memory. True information $h(T=\tau \mid G)$ and $h(T=\tau \mid-G)$ about a group is stored in a decision maker's long term memory. Representative types, being distinctive of the group under consideration, are more readily recalled than other types. As a result, representative types play an outsized role in judgments; other types are relatively neglected.

This setup can be applied to prediction and inference problems (as in BCGS 2016 and BGS 2016). Consider the example from the introduction of a doctor assessing the health status of a patient, $T=\{$ healthy, sick $\}$ in light of a positive medical test, $G=$ positive. The positive test is assessed in the context of untested patients $(-G=$ untested). Applying the previous definition, being sick is representative of patients who tested positive if and only if: 


$$
\frac{\operatorname{Pr}(T=\text { sick } \mid G=\text { positive })}{\operatorname{Pr}(T=\text { sick } \mid-G=\text { untested })}>\frac{\operatorname{Pr}(T=\text { healthy } \mid G=\text { positive })}{\operatorname{Pr}(T=\text { healthy } \mid-G=\text { untested })},
$$

namely when $\operatorname{Pr}(G=$ positive $\mid T=$ sick $)>\operatorname{Pr}(G=$ positive $\mid T=$ healthy $)$. The condition holds if the test is even minimally informative of health status. A positive test brings "sick" to mind because the true probability of this type has increased the most after the positive test is revealed. Thus, the doctor may deem the sick state likely, even if the disease is rare (Casscells et al. 1978), committing a form of base rate neglect described in TK's (1974).

We apply this logic to the problem of forecasting a firm's earnings. The analyst must infer the firm's type $f_{i, t}$ after observing the current earnings surprise $x_{i, t}-b x_{i, t-1}-a \hat{f}_{i, t-1}$. This is akin to seeing the medical test. As we saw previously, the true conditional distribution of firm fundamentals $f_{i, t}$ is normal, with variance $\sigma_{f}^{2}$ and the mean given by Equation (3). This is our target distribution $h(T=\tau \mid G)$. As in the medical example, the information content of the earnings $x_{i, t}$ for fundamentals $f_{i, t}$ is assessed relative to the background information set in which no news is received, namely if the earnings surprise is zero $x_{i, t}-b x_{i, t-1}=a \hat{f}_{i, t-1}$. The comparison distribution $h(T=\tau \mid-G)$ is thus also, normal with mean $a \hat{f}_{i, t-1}$ and variance $\sigma_{f}^{2}$.

With normality, the representativeness of fundamental $f$ for firm $i$ at date $t$ is:

$$
R\left(f, x_{i, t}-b x_{i, t-1}\right)=\exp \left\{\frac{\left(\hat{f}_{i, t}-a \hat{f}_{i, t-1}\right)\left(2 f-a \hat{f}_{i, t-1}-\hat{f}_{i, t}\right)}{2 \sigma_{f}^{2}}\right\} .
$$

If news are good, in the sense that they rationally imply better fundamentals, $\hat{f}_{i, t}>a \hat{f}_{i, t-1}$, representativeness is higher for higher types $f$. After bad news, implying $\hat{f}_{i, t}<a \hat{f}_{i, t-1}$, representativeness is higher for lower types $f$. In the first case, high types are overweighed while low types are underweighted in judgments. In the latter case, the reverse is true.

Appendix A shows that these distortions generate diagnostic beliefs as follows: 
Proposition 1 (Diagnostic Kalman filter) In the long run, upon seeing $x_{i, t}-b x_{i, t-1}$, the analyst's posterior about the firm's fundamentals are normally distributed with variance $\sigma_{f}^{2}$ and mean:

$$
\hat{f}_{i, t}^{\theta}=a \hat{f}_{i, t-1}+K(1+\theta)\left(x_{i, t}-b x_{i, t-1}-a \hat{f}_{i, t-1}\right) .
$$

When analysts overweight representative types, their beliefs resemble the optimal Kalman filter, but with a key difference: they exaggerate the signal to noise ratio, inflating the fundamentals of firms receiving good news and deflating those of firms receiving bad news. Exaggeration of the signal to noise ratio is reminiscent of overconfidence, but here over-reaction occurs with respect to public as well as private news. ${ }^{9}$ The psychology is in fact very different from overconfidence: in our model, as in the medical test example, overreaction is caused by neglect of base rates. After good news, the most representative firms are Googles. This firm type readily comes to mind and the analyst exaggerates its probability, despite the fact that Googles are rare. After bad news, the most representative firms are lemons. The analyst exaggerates the probability of this type, despite the fact that lemons are also quite rare. Exaggeration in the reaction to news increases in $\theta$. At $\theta=0$ the model reduces to rational learning.

The key property of diagnostic expectations is "the kernel of truth": distortions in beliefs exaggerate true patterns in the data. The kernel of truth distinguishes our approach from alternative theories of extrapolation such as adaptive expectations or BSV (1998). As we map the model to the facts of Sections I and II, we first show that the kernel of truth is consistent with the data: Googles are overweighed in the HLTG portfolio because they occur much more often there than elsewhere.

\section{The Model and the Facts}

\footnotetext{
${ }^{9}$ In fact, overconfidence predicts under-reaction to public news such as earnings releases (see Daniel et al. 1998).
} 
To link our model to the data we shift attention from the level of earnings to the growth rate of earnings, which is what analysts predict when they report LTG. Denote by $h$ the horizon over which the growth forecast applies, which is about 4 years for LTG. Define the LTG of firm $i$ at time $t$ as the firm's expected earnings growth over this horizon, namely $L T G_{i, t}=$ $\mathbb{E}_{i, t}^{\theta}\left(x_{t+h}-x_{t}\right)$. By Equations (1) and (6), this boils down to:

$$
L T G_{i, t}=-\left(1-b^{h}\right) x_{t}+a^{h} \frac{1-(b / a)^{h}}{1-(b / a)} \hat{f}_{i, t}^{\theta}
$$

Expectations of long-term growth are shaped by mean reversion in eps and fundamentals. LTG is high when firms have experienced positive news, so $\hat{f}_{i, t}^{\theta}$ is high, and/or when current earnings $x_{t}$ are low, which also raises future growth. Both conditions line up with the evidence, which shows HLTG firms have experienced fast growth (Figure 2), and have low eps (Table 1).

We begin by testing for the kernel of truth. To this end, we first report in Figure 6 the true distribution of future eps growth for the HLTG portfolio (blue curve) against the distribution of future eps growth of all the other firms (orange curve).

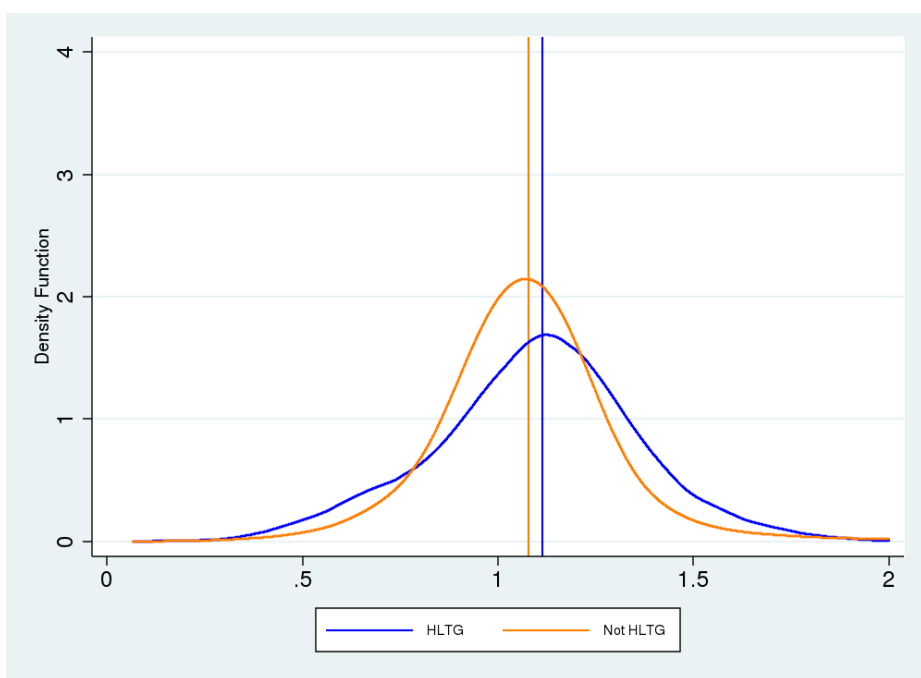


Figure 6 Kernel density estimates of growth in earnings per share for LTG Portfolios. In December of years $(t)$ $1981,1986, \ldots$, and 2011, we form decile portfolios based on ranked analysts' expected growth in long term earnings per share $(L T G)$. For each stock, we compute the gross annual growth rate of earnings per share between $t$ and $t+5$. We exclude stocks with negative earnings in year $t$ and we estimate the kernel densities for stocks in the highest (HLTG) decile and for all other firms with LTG data. The graph shows the estimated density kernels of growth in earnings per share for stocks in the HLTG (blue line) and all other firms (orange line). The vertical lines indicate the means of each distribution (1.11 vs. 1.08, respectively).

Two findings stand out. First, HLTG firms have a higher average future eps growth than all other firms, as we saw in a somewhat different format in Figure 2. Second, and critically, HLTG firms display a fatter right tail of exceptional performers. Googles are thus representative for HLTG in the sense of definition (4). In fact, based on the densities in Figure 6, the most representative future growth realizations for HLTG firms are in the range of $40 \%$ to $60 \%$ annual growth. ${ }^{10}$

In light of these data, our model predicts that analysts should over-estimate the number of right-tail performers in the HLTG group. Figure 7 compares the distribution of future performance of HLTG firms (blue line) with the predicted performance for the same firms (red line). ${ }^{11}$ Consistent with diagnostic expectations, analysts vastly exaggerate the share of exceptional performers, which are most representative of the HLTG group according to the true distribution of future eps growth. ${ }^{12}$ As a robustness check, we reproduce in Appendix C Figures 6 and 7 using as a measure of fundamentals revenues minus cost of goods sold (which may be less noisy that eps). With this metric as well, the evidence supports the kernel of truth hypothesis.

\footnotetext{
${ }^{10}$ Although HLTG firms tend to have also a slightly higher share of low performers, it is true that, as in our model, higher growth rates are more representative for HLTG firms. See Figure C.1 in Appendix C.

${ }^{11}$ In making this comparison, bear in mind that analysts report point estimates of a firm's future earnings growth and not its full distribution (in our model, they report only the mean $f_{i, t}^{\theta}$ and not the variance $\sigma_{f}^{2}$ ). Thus, under rationality the LTG distribution would have the same mean but lower variance than realized eps growth.

12 The kernel of truth can also shed light on the asymmetry between HLTG and LLTG firms. In Appendix C we show that future performance of LLTG firms tends to be concentrated in the middle, with a most representative growth rate of $0 \%$. It is thus constant, rather than bad, performance that is representative of LLTG firms. This fact can help explain why expectations about these firms and their market values are not overly depressed. One could capture this difference between HLTG firms (representative high growth) and LLTG firms (representative 0\% growth) by relaxing the assumption of normality, or alternatively by allowing lower volatility for firms in the LLTG group.
} 


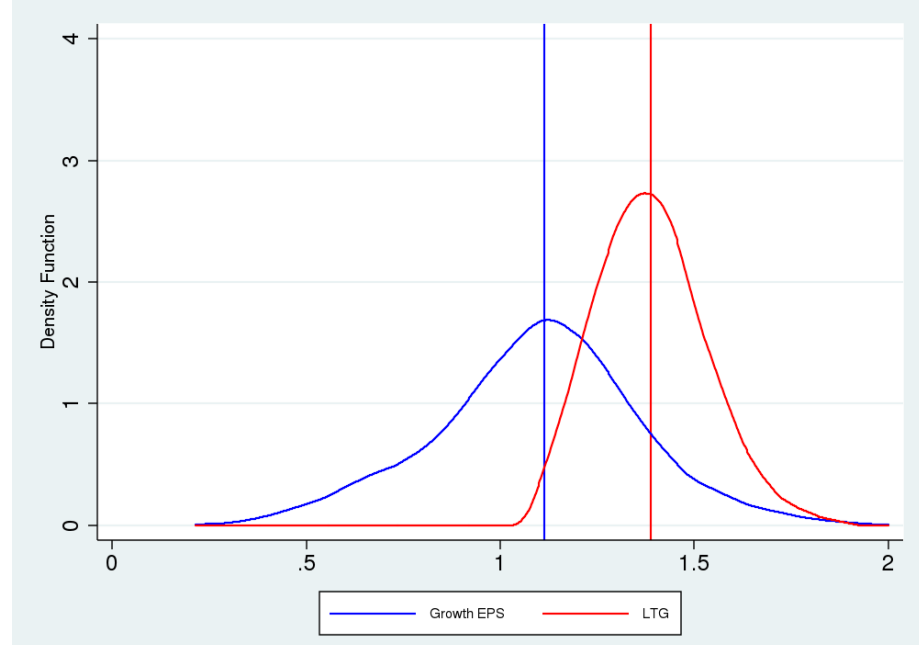

Figure 7. Realized vs. Expected Growth in eps. In December of years $(t)$ 1981, 1986, .., 2006, and 2011, we form decile portfolios based on ranked analysts' expected growth in long term earnings per share (LTG). We plot two series. First, we plot the kernel distribution of the gross annual growth rate in earnings per share between $t$ and $t+5$. Second, we plot the kernel distribution of the expected growth in long term earnings at time $t$. The graph shows results for stocks in the highest decile of expected growth in long term earnings at time $t$. The vertical lines indicate the means of each distribution (1.11 vs. 1.39, respectively).

We next show that the model accounts for the previously documented facts. To explore the dynamics of LTG in our model, we focus on the long run distribution of fundamentals $f_{i, t}$ (which has zero mean and variance $\frac{\sigma_{\eta}^{2}}{1-\alpha^{2}}$ ) and of analysts' mean beliefs $\hat{f}_{i, t}^{\theta}$ (which has zero mean and variance $\left.\sigma_{f}^{2}\right) .{ }^{13}$ In line with our empirical analysis, at time $t$ we identify the high LTG group $\mathrm{HLTG}_{\mathrm{t}}$ as the $10 \%$ of firms with highest believed fundamentals, and hence with highest assessed future earnings growth, and the low LTG group $\mathrm{LLTG}_{\mathrm{t}}$ as the $10 \%$ of firms with lowest believed fundamentals and hence lowest assessed future earnings growth.

\section{V.A. Representativeness and the Features of Expectations}

\footnotetext{
13 There is no distortion in the average diagnostic expectation across firms because in steady state there are no systematic earnings surprises: the average earnings news in the population of firms is zero. As a consequence, the average diagnostic expectation coincides with the average rational expectation. However, diagnostic beliefs are fattertailed than rational ones, because they exaggerate the frequency of Googles and Lemons.
} 
We first review the patterns of fundamentals and expectations documented in Figures 2, 3 and 4. In Section V.B, we review the patterns of returns documented in Figures 1 and 5.

We start from Figure 2, which says that HLTG firms experience a period of pronounced growth before portfolio formation, while LLTG firms experience a period of decline.

Proposition 2. Provided $a, b, K, \theta$ satisfy

$$
b^{h}+a^{h} \frac{1-(b / a)^{h}}{1-(b / a)}\left[\frac{K(1+\theta)-a}{1-a}\right]>1
$$

the average $H_{L T G}\left(L L T G_{t}\right)$ firm experiences positive (negative) earnings growth pre-formation.

In our model, positive earnings surprises have two conflicting effects on long term growth prospects and thus on LTG. On the one hand, they raise estimated fundamentals $\hat{f}_{i, t}^{\theta}$, which enhance future growth. On the other hand, they lower future growth via mean reversion. Condition (7) ensures that the former effect dominates, so that firms with rosy future prospects (HLTG) are selected from those that have experienced good recent performance, while firms with bad prospects (LLTG) are selected from those that have experienced bad recent performance.

The parametric restriction of Condition (7) is more likely to hold the less severe is mean reversion (i.e., when $b$ is close to 1 ) and the larger is the signal to noise ratio $K$. It is also more likely to hold the larger is $\theta$ and for relatively large $a$ (with $a<K(1+\theta)$ ). Note that condition (7) depends on the parameters of the true earnings process because analysts do not mechanically extrapolate past performance. 
Combined with mean reversion of earnings, Proposition 2 accounts for Figure 2, in which HLTG firms experience positive growth pre-formation, which subsequently cools off, while LLTG firms go through the opposite pattern.

We next show that the model can account for the fact documented in Figure 4, namely that expectations for the long term growth of HLTG firms are excessively optimistic.

Proposition 3. If analysts are rational, $\theta=0$, they make no systematic error in predicting the log growth of earnings of $\mathrm{HLTG}_{t}$ and $\mathrm{LLTG}_{t}$ portfolios:

$$
\mathbb{E}\left(x_{t+h}-x_{t}-L T G_{t}^{\theta=0} \mid \mathrm{HLTG}_{t}\right)=\mathbb{E}\left(x_{t+h}-x_{t}-L T G_{t}^{\theta=0} \mid \mathrm{LLTG}_{t}\right)=0 .
$$

Under diagnostic expectations $\theta>0$, in contrast, analysts systematically over-estimate growth in $\mathrm{HLTG}_{t}$ and under-estimate growth in $\mathrm{LLTG}_{t}$ :

$$
\mathbb{E}\left(x_{t+h}-x_{t}-L T G_{t}^{\theta>0} \mid \mathrm{HLTG}_{t}\right)<0<\mathbb{E}\left(x_{t+h}-x_{t}-\operatorname{LTG}_{t}^{\theta>0} \mid \mathrm{LLTG}_{t}\right) .
$$

Under rational expectations, no systematic forecast error can be detected by an econometrician looking at the data (expectations are computed using the true steady state probability measure). Indeed, when $\theta=0$, the average forecast within the many firms of the $\mathrm{HLTG}_{t}$ and LLTG $\mathrm{L}_{t}$ portfolios is well calibrated to the respective means. Diagnostic expectations, in contrast, cause systematic errors. Firms in the $\mathrm{HLTG}_{t}$ group are systematically over-valued: analysts over-react to their pre-formation positive surprises, and form excessively optimistic forecasts of fundamentals. As a consequence, the realized earnings growth is on average below the forecast. Firms in the $\mathrm{LLTG}_{t}$ group are systematically under-valued. As a consequence, their realized earnings growth is on average above the forecast. As noted in Section III, the prediction of systematic pessimism about LLTG firms is not borne out in the data. 
Finally, our model also yields the boom-bust LTG pattern in the HLTG group (and a reverse pattern in the LLTG group) documented in Figure 3. By Proposition 2, the improving preformation forecasts of HLTG firms are due to positive earnings surprises, while the deteriorating pre formation forecasts of LLTG firms are due to negative ones. But the model also predicts post formation reversals in LTG for both groups of firms. To see this, we compare $L T G_{i, t}$ forecasts made at $t$, with forecasts made for the same firm at $t+s$, namely $L T G_{i, t+s}$.

Proposition 4 Under rational expectations, $\theta=0$, we have that:

$$
\mathbb{E}\left(\mathrm{LTG}_{t+s}^{\theta=0} \mid H L T G_{t}\right)-\mathbb{E}\left(\mathrm{LTG}_{t}^{\theta=0} \mid H L T G_{t}\right)<0
$$

Under diagnostic expectations, $\theta>0$, we have that:

$$
\mathbb{E}\left(\mathrm{LTG}_{t+s}^{\theta>0} \mid H L T G_{t}\right)-\mathbb{E}\left(\mathrm{LTG}_{t}^{\theta>0} \mid H L T G_{t}\right)=\mathbb{E}\left(\mathrm{LTG}_{t+s}^{\theta=0} \mid H L T G_{t}\right)-\mathbb{E}\left(\mathrm{LTG}_{t}^{\theta=0} \mid H L T G_{t}\right)-\theta \Psi
$$

for some $\Psi>0$. The opposite pattern, with reversed inequality and $\Psi<0$, occurs for $L L T G_{t}$.

Mean reversion in LTG obtains under rational expectations, due to mean reversion in fundamentals. Under diagnostic expectations, however, mean reversion is amplified by the correction of initial forecast errors. Post-formation, the excess optimism of HLTG firms on average dissipates, causing a cooling off in expectations $\theta \Psi$ that is more abrupt than what would be implied by mean reversion alone. The cooling off of excess optimism arises because there are no news on average in the HLTG portfolio, which on average causes no overreaction. Likewise, the excess pessimism of LLTG firms dissipates, strengthening the reversal in that portfolio. ${ }^{14}$

\footnotetext{
${ }^{14}$ Going forward, firms in the HLTG portfolio receive neither positive nor negative true surprises on average, namely $\mathbb{E}_{t}\left(x_{i, t+1}-b x_{i, t-1}-a \hat{f}_{i, t}\right)=0$. As a result, by Equation (6), their assessed fundamentals on average coincides with the rational value $a \hat{f}_{i, t}$. The same logic triggers mean reversion in the LLTG portfolio.
} 


\section{V.B. The diagnostic Kalman filter and returns}

Consider now the return patterns documented in Figures 1 and 5. To explore the implications of diagnostic expectations for portfolio returns, we take the required return $R>1$ as given. The pricing condition for a firm $i$ at date $t$ is then given by:

$$
\mathbb{E}_{t}^{\theta}\left(\frac{P_{i, t+1}+D_{i, t+1}}{P_{i, t}}\right)=R
$$

so that the stock price of firm $i$ is the discounted stream of expected future dividends as of $t$. We assume that $R$ is high enough that the discounted sum converges. By Equation (8), the equilibrium price at $t$ is $P_{i, t}=\mathbb{E}_{t}^{\theta}\left(P_{i, t+1}+D_{i, t+1}\right) / R$. Using this formula and Proposition 2, we find:

Proposition 5. Denote by $R_{t, P}$ the realized return of portfolio $P=H L T G, L L T G$ at $t$. Then, under the condition of Proposition 2, we have that:

$$
R_{t, H L T G}>R>R_{t, L L T G}
$$

Because firms in the HLTG portfolio receive positive news before formation (Proposition 2), they earn returns higher than the required return $R$. Our model thus yields positive abnormal preformation returns for HLTG stocks, as well as the low pre-formation returns of LLTG stocks, as in Figure 5. This effect does not rely on representativeness, as it arises also for $\theta=0$. The key implication of representativeness is predictability of post-formation returns (Figures 1 and 5).

To see this, note that the average realized return going forward (according to the true probability measure) for a given firm $i$ :

$$
\mathbb{E}_{t}\left(\frac{P_{i, t+1}+D_{i, t+1}}{P_{i, t}}\right)=\frac{\mathbb{E}_{t}\left(P_{i, t+1}+D_{i, t+1}\right)}{\mathbb{E}_{t}^{\theta}\left(P_{i, t+1}+D_{i, t+1}\right)} R .
$$


The average realized return at $t+1$ is below the required return $R$ when at $t$ investors over-value the future expected price and dividend of firm $i$, namely when the denominator is larger than the numerator in Equation (9). Conversely, the average realized return at $t+1$ is higher than the required return $R$ when at $t$ investors under-value the firm's future expected price and dividend.

Diagnostic expectations thus yield the return predictability patterns of Figure 5.

Proposition 6. (Predictable Returns) Denote by $\mathbb{E}_{t}\left(R_{t+1}^{\theta} \mid P\right)$ the average future return of portfolio $P=$ HLTG, LLTG at $t+1$. Under rationality, $\theta=0$, excess returns are not predictable:

$$
\mathbb{E}_{t}\left(R_{t+1}^{\theta=0} \mid H L T G\right)=R=\mathbb{E}_{t}\left(R_{t+1}^{\theta=0} \mid L L T G\right)
$$

Diagnostic expectations generate predictable excess returns:

$$
\mathbb{E}_{t}\left(R_{t+1}^{\theta>0} \mid H L T G\right)<R<\mathbb{E}_{t}\left(R_{t+1}^{\theta>0} \mid L L T G\right)
$$

Under rational expectations (i.e., for $\theta=0$ ) realized returns may differ from the required return $R$ for particular firms. However, the rational model cannot account for systematic return predictability in a large portfolio of firms sharing a certain forecast. Conditional on current information, rational forecasts are on average (across firms) correct and returns are unpredictable.

Under the diagnostic Kalman filter $(\theta>0)$, in contrast, the HLTG portfolio exhibits abnormally high returns up to portfolio formation and abnormally low returns after formation. The converse holds for the LLTG portfolio, just as we saw in Table 1 and Figure 1. This is because post-formation expectations systematically revert to fundamentals, and in particular investors are systematically disappointed in HLTG firms and their returns are abnormally low. 
To summarize, the return predictability documented in Figure 1 can be accounted by representativeness but not by rational learning. Rational learning can account for the preformation return patterns (Proposition 5), but it does not yield post formation reversals (Proposition 6) whereby HLTG stocks underperform LLTG stocks.

\section{Additional Predictions of the Model}

Diagnostic expectations yield a coherent account of the dynamics of news, analyst expectations, and returns documented in Section III. Three issues remain open. The first is whether analyst expectations (and hence returns) indeed over-react to news. The challenge of testing this hypothesis is that news are hard to measure, as they include but are not restricted to observed earnings. The second issue is whether our model can account quantitatively, and not only qualitatively, for the dynamics of analyst expectations and returns of Section III. A third issue is whether the diagnostic Kalman filter improves upon existing theories of non-rational expectations such as BSV's (1998) model of investor sentiment or adaptive expectations.

This section addresses these issues by highlighting additional implications of our model. We assess the role of over-reaction in Section VI.A, the quantitative performance of the model in Section VI.B, and alternative models of expectations formation in Section VI.C. In this last section, we also revisit the possibility that analysts may be learning rationally from prices about investors' time varying required returns. As we noted in Section III, this theory does not account for expectations errors, but it could account for return predictability.

\section{VI.A Overreaction to News and Returns}


To assess over-reaction to news, we first need to have a measure of news. Coibion and Gorodnichenko (2015) propose measuring the news received by the forecaster at time $t$ using their forecast revision at $t$. Such revision can in fact be interpreted as a summary for all information received by the forecaster in the recent past. Forecasters' over or under reaction to information can then be assessed by correlating their forecast revision with the subsequent forecast error.

In particular, Coibion and Gorodnichenko show that in sticky information or rational inattention models (Sims 2003, Mankiw and Reis 2002), consensus forecast revisions should positively correlate with subsequent consensus forecast errors. Intuitively, when expectations under-react, a positive forecast revision indicates insufficient upward adjustment. As a result, it should predict positive errors (i.e. realizations above the forecast). Bouchaud et al. (2016) use this method to diagnose under-reaction to news about firms' profitability. The same approach turns out to be useful for our analysis as well.

Proposition 7. Assume the condition (7) of Proposition 2. Consider the firm level regression

$$
x_{i, t+h}-x_{i, t}-L T G_{i, t}=\alpha+\gamma\left(L T G_{i, t}-L T G_{i, t-k}\right)+v_{i, t+h}
$$

Under rationality, $\theta=0$, the estimated $\gamma$ is zero, while it is negative for $\theta>0$.

If analysts overreact - namely $\theta>0$ - as predicted by diagnostic expectations, an upward revision in LTG is symptomatic of excessive adjustment, which in turn predicts a negative forecast error (LTG above realized growth), namely $\gamma<0$. In contrast, under rational expectations, forecast errors should be unpredictable at the individual level. ${ }^{15}$

\footnotetext{
${ }^{15}$ A positive estimate of $\gamma$ would suggest, in our model, that analysts discount highly representative types. This is equivalent to having a negative $\theta$ in Equation (5).
} 
Table 2 below reports the estimates from the univariate regression of forecast error, defined as the difference between average growth over $h=3,4,5$ years and current LTG, and the revision of LTG over the past $k=1,2,3$ years. We allow horizon $h$ to vary because LTG refers to growth over a period between 3 to 5 years. To estimate $\gamma$ we use consensus forecasts rather than individual analyst estimates because many analysts drop out of the sample. ${ }^{16}$

\section{Table 2: Coibion-Gorodnichenko regressions for EPS}

Each entry in the table corresponds to the estimated coefficient of the forecast errors $\left(\mathrm{eps}_{\mathrm{t}+\mathrm{n}} / \mathrm{eps} \mathrm{t}_{\mathrm{t}}\right)^{1 / \mathrm{n}}-\mathrm{LTG}_{\mathrm{t}}$ for $\mathrm{n}=3,4$, and 5 on the variables listed in the first column of the table and year fixed-effects (not shown).

\begin{tabular}{l|ccc}
\multicolumn{4}{c}{ Dependent Variable } \\
\multicolumn{1}{c}{} & $\left(\mathrm{eps}_{\mathrm{t}+3} / \mathrm{eps}_{\mathrm{t}}\right)^{1 / 3}-\mathrm{LTG}_{\mathrm{t}}$ & $\left(\mathrm{eps}_{\mathrm{t}+4} / \mathrm{eps}_{\mathrm{t}}\right)^{1 / 4}-\mathrm{LTG}_{\mathrm{t}}$ & $\left(\mathrm{eps}_{\mathrm{t}+5} / \mathrm{eps}_{\mathrm{t}}\right)^{1 / 5}-\mathrm{LTG}_{\mathrm{t}}$ \\
\hline $\mathrm{LTG}_{\mathrm{t}}-\mathrm{LTG}_{\mathrm{t}-1}$ & -0.0351 & $-0.1253^{\mathrm{c}}$ & $-0.1974^{\mathrm{a}}$ \\
& $(0.0734)$ & $(0.0642)$ & $(0.0516)$ \\
& & & $-0.2930^{\mathrm{a}}$ \\
$\mathrm{LTG}_{\mathrm{t}}-\mathrm{LTG}_{\mathrm{t}-2}$ & $-0.2335^{\mathrm{a}}$ & $-0.2687^{\mathrm{a}}$ & $(0.0452)$ \\
& $(0.0625)$ & $(0.0602)$ & \\
& & & $-0.3127^{\mathrm{a}}$ \\
$\mathrm{LTG}_{\mathrm{t}}-\mathrm{LTG}_{\mathrm{t}-3}$ & $-0.2897^{\mathrm{a}}$ & $-0.2757^{\mathrm{a}}$ & $(0.0437)$ \\
& $(0.0580)$ & $(0.0565)$ & \\
\hline
\end{tabular}

Consistent with diagnostic expectations, upward LTG revision predicts excess optimism, pointing to over-reaction to news. This holds regardless of the forecast horizon $h$, so the pattern is robust to alternative interpretations of LTG. The estimated $\gamma$ tends to become more negative and more statistically significant at longer forecast horizons $h=3,4,5$ (i.e. as we move from left to right in Table 2), perhaps reflecting the difficulty of projecting growth into the future. ${ }^{17}$

\footnotetext{
16 Estimating (10) on the consensus LTG may misleadingly indicate under-reaction if individual analysts observe noisy signals, so that there is dispersion in their forecasts. Coibion and Gorodnichenko show that when different analysts observe noisy signals, the coefficient in Equation (10) is positive even if each analyst rationally revises his forecast. In this respect, finding negative $\gamma$ in a consensus regression is even stronger evidence of over-reaction to information.

17 This feature helps reconcile our evidence with the sluggishness documented by Bouchaud et al. (2016). They consider forecasts for the level of eps over short horizons such as 1 or 2 years. In Appendix D (see Table D.2) we
} 
Interestingly, the estimated $\gamma$ also gets higher in magnitude and more statistically significant as we lengthen the revision period $k=1,2,3$ (i.e. moving from top to bottom in Table 2). We view this evidence as being consistent with the kernel of truth. From Equation (6), overreaction to information of the diagnostic filter $\hat{f}_{i, t}^{\theta}$ compared to the rational filter $\hat{f}_{i, t}$ is given by:

$$
\hat{f}_{i, t}^{\theta}-\hat{f}_{i, t}=K \theta\left(x_{i, t}-b x_{i, t-1}-a \hat{f}_{i, t-1}\right)
$$

where $K$ is the true signal to noise ratio of information accruing during the revision period. It is plausible that persistent signals over 2 or 3 years are objectively more informative about future earnings, i.e. have a higher $K$, than occasional signals accruing over one year. By the kernel of truth, then, such signals should induce more over-reaction, consistent with the data.

To the extent that over-reaction drives excess optimism about HLTG, and thus lower post formation returns, stronger over-reaction should be associated with larger return spreads between HLTG and LLTG portfolios. In Appendix D.3 we show that industries in which overreaction is larger (i.e. $\gamma$ is larger) also feature larger return spreads. While these results should be taken with caution due to the small number of industries, they are suggestive of a direct link between overreaction and predicable returns.

\footnotetext{
show that at these horizons there is some evidence of under-reaction also in our data (with the usual caveat of the under-reaction bias entailed in estimating consensus regressions). The seemingly contradictory findings of over and under-reaction can be reconciled by combining diagnostic expectation with some short run rigidity in analyst forecasts, stemming for instance from sporadic revision times. In this case, a piece of news would initially trigger few adjustments, generating short term aggregate under-reaction, but will lead to overreaction as all analysts update.
} 
Overall, this subsection offers evidence in support of the hypothesis that expectation formation about LTG features over-reaction to news, consistent with diagnostic expectations, but inconsistent with rational inattention or other theories of under-reaction. ${ }^{18}$

\section{VI.B Model Calibration}

We now provide a back of the envelope calibration of our model. Among other things, this exercise yields an estimate of the strength $\theta$ of representativeness, quantifying the extent of departures from rationality in expectations and returns.

The dynamics of firm level earnings and expectations depend on five parameters: the persistence and conditional variance of observed log earnings per share ( $b$ and $\sigma_{\epsilon}$ from Equation 1), those of fundamentals $f$ ( $a$ and $\sigma_{\eta}$ from Equation 2), and the strength of representativeness $\theta$. To predict returns, we also need to pin down the value of the required rate of return $R$.

We set the five parameters $\left(a, b, \sigma_{\eta}, \sigma_{\epsilon}, \theta\right)$ to match the autocorrelation of earnings per share of order 1, 2, 3, and 4, and the coefficient $\gamma$ estimated in Section VI.A linking forecast error $\left(\frac{e p s_{t+4}}{e p s_{t}}\right)^{1 / 4}-L T G_{t}$ to forecast revision $L T G_{t}-L T G_{t-3} \cdot{ }^{19}$

We fix a parameter combination $\left(a, b, \sigma_{\eta}, \sigma_{\epsilon}, \theta\right)$, simulate the model, and compute the implied value for the five moments we seek to match. This yields the vector:

\footnotetext{
18 The evidence of the over-reaction to news of consensus LTG forecasts is also inconsistent with theories of overreaction based on analyst overconfidence such as Daniel et al. (1998). In these models, analysts over-react to their private information but under-react to common information, generating under-reaction of consensus forecasts.

${ }^{19}$ The parameters of the model could in principle be estimated by fitting a Kalman filter to the data of individual firms, but this is hampered by the fact that the time series of annual data is short, and can have negative earnings (which are assumed away in the model). For this reason, we calibrate the model by matching moments of the pooled data. We estimate autocorrelation coefficients by pooling all the observations in our dataset and running univariate OLS regressions of log earnings on its lagged value.
} 


$$
v\left(a, b, \sigma_{\eta}, \sigma_{\epsilon}, \theta\right)=\left(\hat{\rho}_{1}, \hat{\rho}_{2}, \hat{\rho}_{3}, \hat{\rho}_{4}, \hat{\gamma}\right)
$$

where $\hat{\rho}_{l}=\frac{\operatorname{cov}\left(x_{t}, x_{t-l}\right)}{\operatorname{var}\left(x_{t}\right)}$ is the model implied autocorrelation of (log) earnings of order $l$ (years), and $\hat{\gamma}$ is coefficient obtained by estimating Equation (10) using the data generated by the model under the same parameter combination.

We repeat the above exercise for each parameter combination $\left(a, b, \sigma_{\eta}, \sigma_{\epsilon}, \theta\right)$ in a grid defined by $a, b \in[0,1], \sigma_{\eta}, \sigma_{\epsilon} \in[0,0.5]$ and $\theta \in[0,3]$ (in steps of 0.1 ). We calibrate the parameters by picking the combination that minimizes the Euclidean distance loss function

$$
\ell(v)=\|v-\bar{v}\|
$$

where $\bar{v}$ is the vector of target moments estimated from the pooled data of all firms, given by:

$$
\bar{v}=(0.82,0.75,0.70,0.65,-0.282) \text {. }
$$

The table below reports the average and standard deviation of the ten parameter combinations that yield the lowest value of the Euclidean loss function.

Table 3: Calibration of model parameters

\begin{tabular}{|c|c|c|c|c|}
\hline$a$ & $b$ & $\sigma_{\eta}$ & $\sigma_{\epsilon}$ & $\theta$ \\
\hline 0.90 & 0.33 & 0.15 & 0.17 & 1.22 \\
$(0.01)$ & $(0.07)$ & $(0.05)$ & $(0.05)$ & $(0.18)$ \\
\hline
\end{tabular}

The high value of $a$ means that fundamentals are estimated to be persistent. The relatively low value of $b$ then implies that shocks to log earnings mean revert fast. The variance of fundamentals $\sigma_{\eta}$ and of transitory earnings $\sigma_{\epsilon}$ are estimated to be similar. As a first sanity check 
for our calibrated log-earnings process, we can compare it to the estimates of the persistence of earnings from the large accounting literature. This literature typically fits AR(1) processes for log earnings to the data, and finds that estimates of the auto-regressive coefficient range from 0.77 to 0.84 with a mode at 0.8 (Sloan 1996). If we fit an AR(1) to the data simulated with the calibrated parameters, we estimate a persistence coefficient of 0.82 , very close to its empirical counterpart.

Our calibration yields a positive $\theta$, which entails over-reaction to news, and the value of 1.22 is fairly close to the estimate for the same parameter obtained by BGS (2017) in the context of credit spreads $(\theta=0.91)$. A $\theta$ of the order of one intuitively implies that the magnitude of forecast errors is comparable to the magnitude of news (i.e., in the current context, it implies a doubling of the signal to noise ratio).

Finally, we calibrate the required rate of return on stocks to $R=9.7 \%$, which is the historical value-weighted average market return. Using these six calibrated parameters, we reproduce and report in Figure 8 the simulated versions of Figures 1 through 6. 

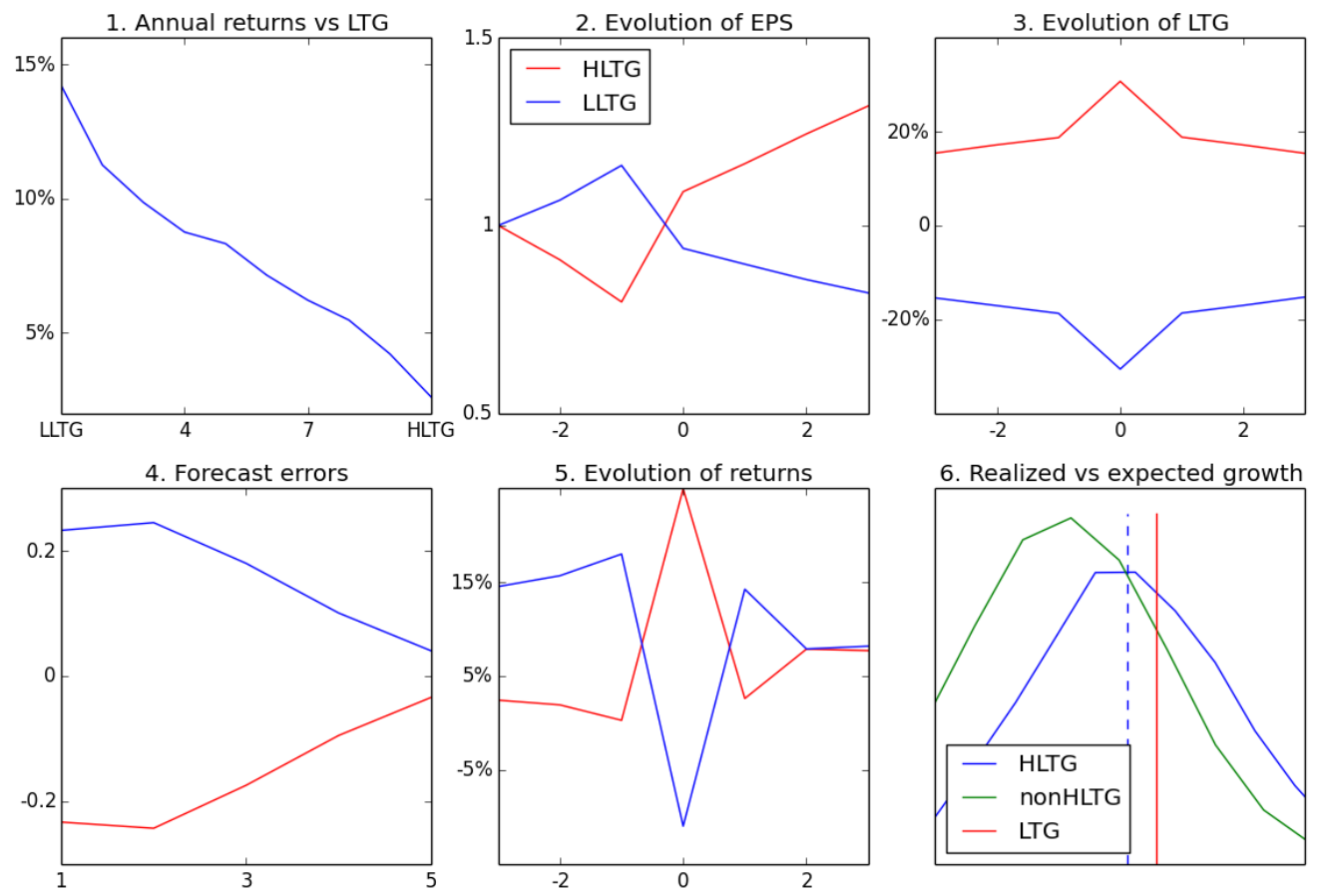

Figure 8. Simulation of the calibrated model. Using the parameters in Table 3 , and $R=9.7 \%$, we simulate 4000 firms over 100 time periods, generating time series of fundamentals, earnings, and growth expectations. At each time period $t$, we sort firms on LTG forecasts. Panel 1 shows the average return spread 1 year post-formation across LTG deciles. We first compute, for each period $t$, the arithmetic average return for each portfolio. We then compute the geometric average of portfolio returns over time. Panels 2, 3, and 5 show the average EPS, LTG, and returns of the HLTG and LLTG portfolios from year $t-3$ to year $t+3$. Panel 4 shows the forecast error on HLTG and LLTG portfolios in the 5 years porst-formation. Panel 6 shows the distribution of realized earnings growth after 5 years for HLTG and for non-HLTG firms, together with the forecast for growth after 5 years.

The model reproduces the main qualitative features of the data. In Panel 1, it reproduces the return spread between HLTG and LLTG stocks. In Panel 2, it reproduces the pattern of Figure 2 that pre-formation HLTG firms have fast growth, which then declines post formation. In Panel 3 the model reproduces the boom bust dynamics of LTG, with analysts' expectations becoming more optimistic pre-formation, and then reverting post-formation. Panel 4 reproduces the finding of Figure 4 of large forecast errors (excess optimism) for HLTG stocks. ${ }^{20}$ Panel 5 reproduces the

${ }^{20}$ The simulation produces forecasts at all horizons, so Panel 4 shows the forecast error in each year from $t+1$ to $t+$ 5 . In contrast, Figure 4 plots the difference between the realized growth in those years and the LTG forecast in year $t$. 
boom-bust pattern in returns around portfolio formation. Returns for HLTG stocks are very high pre-formation, but then collapse below the required return $R$ in the immediate post formation period, and eventually reverting back to their unconditional, long term value. The opposite happens to the return of LLTG stocks. Finally, and crucially, the model exhibits the kernel of truth, in that HLTG firms do perform better going forward than non-HLTG firms (panel 6). ${ }^{21}$

The model fails to capture some qualitative features of the data. It does not capture the persistent high earnings growth of HLTG stocks before formation, nor the negative forecast error for LLTG. We return to these issues when we summarize the results of the calibration.

We now assess how the model performs quantitatively. Consider first the cross sectional predictability of returns. The calibrated model entails an average LLTG-HLTG yearly return spread of $15 \%$ in year $t+1$ (see Panel 1). At the portfolio level, in the calibration LLTG earns average yearly returns of $20 \%$ while HLTG earns $5 \%$. The empirical counterparts to these values are $15 \%$ and $3 \%$, with a gap of $12 \%$. Thus, a calibration based on earnings and expectations data provides a good match to the evidence on returns. ${ }^{22}$

We can also assess model performance regarding the dynamics of expectations, relative to the underlying earnings process. In doing so, we note upfront that the annualized levels of earnings growth (and expectations thereof) over a four year horizon obtained in the model are roughly one-fourth the size of their empirical counterparts. Despite this level effect, which we revisit below, the dynamics of the simulation provide a reasonable match to the data on expectations. For example, the average LTG for HLTG firms at formation is 3.25 times their

\footnotetext{
${ }^{21}$ As a check, we show in the Appendix that imposing rational expectations $(\theta=0)$ yields zero average forecast errors and average returns equal to the required return for all portfolios.

${ }^{22}$ In the Appendix, we check the robustness of this quantitative performance as a function of $\theta$ (keeping the other parameters fixed). Figure D.2 shows that the match with the HLTG-LLTG return spread (Figure 1) is best at $\theta=1.2$, and decays strongly as $\theta$ deviates from this value.
} 
actual EPS annual growth rate over the subsequent 4 years (39\% vs $12 \%$, see Figure 7$)$. In our model, LTG exaggerates growth by a factor of $2.14(7.5 \%$ vs $3.5 \%)$.

The model also captures both the size and the speed of the boom-bust pattern in expectations: from year $t-3$ to year $t+3$, simulated LTG forecasts for HLTG firms rise by $100 \%$ relative to baseline and then fall again. In the data, the corresponding figure is $68 \%$. Importantly, in both the model and the data, the bulk of action happens in years $t-1$ to $t+1 .^{23}$

Our overall assessment is that the model is able, both qualitatively and quantitatively, to account for several key features of the data, including the predictable return spread between HLTG and LLTG portfolios and the dynamics of expectations relative to earnings process. At the same time, the model is very stylized, abstracting away from both firm and investor heterogeneity. These assumptions can be relaxed without compromising the tractability of the Diagnostic Kalman filter. An appropriate treatment of firm heterogeneity and variation in beliefs would likely help in accounting for the features our model does not capture.

For example, the model fails to reproduce the pre-formation EPS dynamics of Figure 2, and the pre-formation return dynamics of Figure 5. In fact, HLTG firms experience strong persistent growth (and positive returns) in years $t-3$ through $t$. However, allowing for firm heterogeneity would improve the fit, because HLTG firms are disproportionately younger and thus smaller than average. ${ }^{24}$ The same mechanism would generate much higher growth (and expectations thereof) for HLTG firms, yielding a better match also with the growth levels in the

\footnotetext{
${ }^{23}$ As noted in Section III, the model predicts a positive forecast error for LLTG portfolio, which is not true in the data.

${ }^{24}$ In our calibration, high LTG is associated with low EPS, because mean reversion is strong ( $b$ is low). In steady state, this requires negative growth, and poor returns, in the pre-formation years $t-3$ to $t-1$, as in Figure 8 . But in reality, HLTG are disproportionately young firms that are starting out small. For such firms, persistent growth would in fact lead to high growth forecasts. Thus, accounting for heterogeneity in firm age would improve the match with the data, and also capture asymmetries in performance between HLTG and LLTG firms.
} 
data. In turn, accounting for heterogeneity in investors' beliefs would capture the dispersion in LTG forecasts, which we abstract from here. In Figure 1, the return spread is strongest for the highest deciles of the LTG distribution, but is shallower in the lower deciles, particularly after 1998 (see Figure B.1 in Appendix B). This is consistent with the possibility that, since 1998, arbitrage improved for LLTG firms but not for HLTG firms, which are smaller and more costly to arbitrage.

\section{VI.C Alternative Mechanisms for Overreaction}

We conclude by comparing diagnostic expectations with alternative models of expectation formation. We begin with models of overreaction to news, such as the BSV model of investor sentiment and mechanical extrapolation. BSV is an early attempt to formalize the psychology of representativeness. It assumes that the true process driving a firm's earnings is a random walk, but

analysts perform Bayesian updating across two incorrect models, one where earnings are believed to trend and one where they mean revert. Over-reaction occurs because periods of fast earnings growth induce the analyst to attach a high probability that the firm is of the "trending type", even though no firm is actually trending.

Our model captures the key intuition of BSV: after good performance analysts place disproportionate weight on strong fundamentals, and the reverse after bad performance. It has, however, two main advantages relative to its antecedent. First, in the BSV model extrapolation follows from belief in models. In contrast, our model yields the kernel of truth: the HLTG group features a relatively higher share of Googles, and analysts exaggerate this share in their assessment. This means, in contrast to the above, that belief distortions can be predicted from the data. Figures 6 and 7 are indeed consistent with this prediction. The second advantage of our 
model is portability: it is not designed for a specific finance setting, and so it can be easily applied to probability judgments, learning contexts or stereotyping.

The other conventional approach to over-reaction, mechanical extrapolation, implies that LTG is formed as a distributed lag of past earnings growth rates, following the adaptive rule:

$$
x_{t+1}^{a d}=x_{t}^{a d}+\mu\left(x_{t}-x_{t}^{a d}\right)
$$

where $x_{t}^{a d}$ is the expectation held at $t$ about the level or growth of eps at a certain period, $x_{t}$ is the current realized level of growth of earnings, and $\mu \in[0,1]$ is a fixed coefficient. If $\mu$ is low, expectations under-react to news. But if $\mu$ is large relative to the persistence of the earnings process, expectations can over-react to news.

The difference between our model and mechanical extrapolation is that diagnostic expectations are forward looking. Under the mechanical rule of Equation (11), analysts revise growth expectations downward if and only if bad news arrive, namely if $\left(x_{t}-x_{t}^{a d}\right)<0$. In contrast, under diagnostic expectations decision makers are influenced by the features of the data generating process such as the true share of Googles and the mean reversion of earnings. For instance, when considering firms that have grown fast in the past, such as HLTG ones, growth forecasts will cool off over time even if no news is received.

In fact, the revision of believed fundamentals from one period to the next is given by:

$$
\begin{gathered}
\hat{f}_{i, t+1}^{\theta}-\hat{f}_{i, t}^{\theta}=K(1+\theta)\left(x_{i, t+1}-b x_{i, t}-a \hat{f}_{i, t}^{\theta}\right) \\
-(1-a) \hat{f}_{i, t}-K \theta(1-a K(1+\theta))\left(x_{i, t}-b x_{i, t-1}-a \hat{f}_{i, t-1}\right)
\end{gathered}
$$

The revision depends in part on the surprise relative to diagnostic expectations, namely on $x_{i, t+1}-b x_{i, t}-a \hat{f}_{i, t}^{\theta}$. But even in the absence of surprising earnings, namely when $x_{i, t}-$ $b x_{i, t-1}=a \hat{f}_{i, t-1}$, beliefs about fundamentals are updated. This is partially due to mean reversion 
of fundamentals (i.e. the second term $\left.-(1-a) \hat{f}_{i, t}\right)$, but also due to the waning of over-reaction to previous shocks (i.e. the third term $x_{i, t}-b x_{i, t-1}-a \hat{f}_{i, t-1}$ ). For HLTG stocks, both forces point toward a downward revision of believed fundamentals, regardless of the current news received, while for LLTG stocks the opposite holds. This leads to systematic mean reversion of LTG estimates for these portfolios. In contrast, no systematic mean reversion should be expected under adaptive expectations.

To test this prediction, we consider the change in LTG around earnings announcement dates. We rank earnings of all firms surprises into deciles and follow LTG revisions for each decile. The results are reported in Figure 9 below.

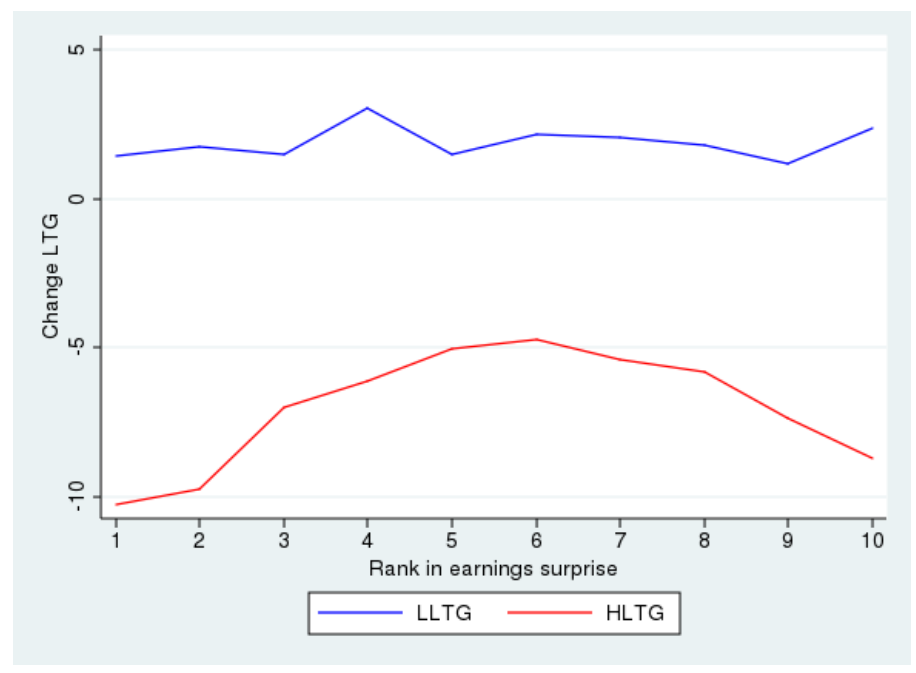

Figure 9. Evolution of Analysts' Beliefs in Response to Earnings' Announcements. For each analyst $j$, firm $i$, and fiscal year $t$, we compute the difference between the first-available LTG forecast made during the 4590 day window following the earnings' announcement for $t-1$ and the first-available LTG forecast made during the 45-90 day window following the earnings' announcement for $t$. We rank observations into deciles based on the ratio of the forecasting error for earnings per share in year $t$ to the stock price when that forecast was made. We measure forecasting errors using the first-available forecasts for earnings per share during the 45-90 day window following the earnings' announcement for $t-1$. The Figure reports the sample average for all observations, for portfolios HLTG and LLTG.

The data show strong evidence of systematic mean reversion. Regardless of the experienced earnings surprise, expectations about HLTG firms deteriorate while those about 
LLTG ones improve. Thus, the reversal of Figure 3 is not simply due to the fact that HLTG firms on average receive bad surprises and LLTG firms on average receive good surprises. Rather, even HLTG firms that experience positive earnings surprises are downgraded, and even LLTG firms that experience negative earnings surprises are upgraded. These findings are puzzling from the perspective of adaptive expectations, but are consistent with the forward-looking nature of diagnostic expectations. $^{25}$ In Appendix E, we show that the same pattern emerges in our calibrated model. ${ }^{26}$

We conclude this section with a discussion of an alternative mechanism of expectation formation compatible with rational expectations, namely the possibility that analyst expectations are formed, at least in part, by rational learning from prices. Suppose that investors' required return is unobservable and follows a mean-reverting process. In this model, price increases signal an improvement in fundamentals but also a decrease in investors' required return (i.e. prices go up partially because the market is bullish about a firm). As a consequence, this mechanism predicts that, for analysts who infer about fundamentals from stock prices, expectations of earnings growth and expectations of returns should be negatively correlated.

To test this prediction, we construct a measure of analysts' expectations of returns by gathering IBES data on the projected price level forecasted by analysts within a 12-month horizon. Historical data on target prices is available since March 1999. We define target returns

\footnotetext{
${ }^{25}$ In the Appendix, we show that adaptive expectations predict no over-reaction to news after the persistence of the earnings process is accounted for. After controlling for current levels $x_{t}$, the adaptive forecast revision $\left(x_{t+1}^{a}-x_{t}^{a}\right)$ should positively predict forecast errors as in the under-reaction models. In contrast, diagnostic expectations overreact to news regardless of the persistence of the data generating process.

${ }^{26}$ Fuster, Laibson and Mendel (2010) suggest an alternative mechanism of expectations formation, natural expectations, according to which investors simplify hump-shaped stochastic processes by neglecting the impact of shocks in the distant past, e.g. by fitting an AR(1). In this model, investors who witness good performance expect growth to continue and exhibit extrapolation. However, earnings processes are reasonably represented by AR(1) processes, which limits the impact of such a simplification mechanism.
} 
as the ratio of the mean target price across all analysts following the stock to the current stock price.

Using this measure, the correlation between analysts' expectations of long-term growth (LTG) and their expected returns is 0.23 (significant at the $1 \%$ level), in contrast to the prediction above. Our model does not provide a meaningful counterpart to this finding, as (diagnostic) expectations of returns are constant and equal to $R$.

\section{Conclusion.}

This paper revisits what since Shiller (1981) has been perhaps the most basic challenge to rational asset pricing, namely over-reaction to news and the resulting excess volatility and mean reversion. We investigate this phenomenon in the context of individual stocks, for which we have extensive evidence on security prices, fundamentals, but also -- crucially -- expectations of future fundamentals. LaPorta (1996) has shown empirically that securities whose long-term earnings growth analysts are most optimistic about earn low returns going forward. Here we propose a theory of belief formation that delivers this finding, but also provides a characterization of joint evolution of fundamentals, expectations, and returns that can be taken to the data.

A central feature of our theory is that investors are forward looking, in the sense that they react to news. However, their reaction is distorted by representativeness, the fundamental psychological principle that people put too much probability weight on states of the world that the news they receive is most favorable to. In psychology, this is known as the kernel of truth hypothesis: people react to information in the right direction, but too strongly. We call such belief formation diagnostic expectations, and show that a theory of security prices based on this model 
of beliefs can explain not just previously documented return anomalies, but also the joint evolution of fundamentals, expectations, and returns.

The theory is portable in the sense that the same model of belief distortions has been shown to work in several other contexts. At the same time, the model can be analyzed using a variation of Kalman Filter techniques used in models of rational learning. Most important, the theory yields a number of strong empirical predictions, which have not been considered before, but which we have brought to the data. Although some puzzles remain, the evidence is supportive of the proposed theory.

Of course, this is just a start. Our approach to expectation formation can be taken to other contexts, most notably aggregate stock prices but also macroeconomic time series. We have focused on distortions of beliefs about the means of future fundamentals, but the kernel of truth idea could be applied to thinking about other moments as well, such as variance or skewness. We hope to pursue these ideas in future work, but stress what we see as the central point: the theory of asset pricing can incorporate fundamental psychological insights while retaining the rigor and the predictive discipline of rational expectations models. And it can explain the data not just on the joint evolution of fundamentals and security prices, but also on expectations, in a unified dynamic framework. Relaxing the rational expectations assumption does not entail a loss of rigor; to the contrary it allows for a disciplined account of additional features of the data. A calibration exercise suggests, moreover, that the model can replicate several quantitative, and not just qualitative, features of the data. 


\section{References}

Adam, Klaus, and Albert Marcet, and Juan Pablo Nicolini. 2016. "Stock Market Volatility and Learning." Journal of Finance 71(1): 33 - 82.

Barberis, Nicholas, and Andrei Shleifer. 2003. "Style Investing." Journal of Financial Economics 68: 161-199.

Barberis, Nicholas, Andrei Shleifer, and Robert Vishny. 1998. "A Model of Investor Sentiment." Journal of Financial Economics 49 (3): 307-343.

Barberis, Nicholas, Robin Greenwood, Lawrence Jin, and Andrei Shleifer. 2015. "X-CAPM: An Extrapolative Capital Asset Pricing Model.” Journal of Financial Economics 115 (1): 1-24.

Barsky, Robert and J. Bradford DeLong. 1993. "Why Does the Stock Market Fluctuate?" Quarterly Journal of Economics 108(2): 291-311.

Ben David, Yitzhak, John Graham, and Campbell Harvey. 2013. "Managerial Miscalibration." Quarterly Journal of Economics 128 (1), 547-84.

Bordalo, Pedro, Katherine Coffman, Nicola Gennaioli, and Andrei Shleifer. 2016. "Stereotypes." Quarterly Journal of Economics 131 (4): 1753-1793.

Bordalo, Pedro, Nicola Gennaioli, and Andrei Shleifer. 2016. "Diagnostic Expectations and Credit Cycles." Journal of Finance, forthcoming.

Bouchaud, Jean-Philippe, Philipp Krueger, Augustin Landier, and David Thesmar. 2016. "Sticky Expectations and the Profitability Anomaly." Working paper.

Casscells, Ward, Arno Schoenberger, and Thomas Graboys. 1978. "Interpretation by Physicians of Clinical Laboratory Results.” New England Journal of Medicine 299 (18):999-1001.

Coibion, Olivier and Yuriy Gorodnichenko 2015. "Information Rigidity and the Expectations Formation Process: A Simple Framework and New Facts" American Economic Review 105(8): 2644-2678.

Cutler, David, James Poterba, and Lawrence Summers. 1990. "Speculative dynamics and the role of feedback traders." American Economic Review 80 (2):63-68.

—. 1991. "Speculative dynamics.” Review of Economic Studies 58 (3): 529-46.

Daniel, Kent, David Hirshleifer, and Avanidhar Subramanyam. 1998. "Investor Psychology and Security Market Under- and Over-Reaction.” Journal of Finance 53(6): 1839 - 1885.

Daniel, Kent, Alexander Klos, and Simon Rottke. 2017. “Overpriced Winners.” Working paper. 
De Bondt, Werner and Richard Thaler. 1985. "Does the Stock Market Overreact?" The Journal of Finance 40(3), 793-805.

De Bondt, Werner and Richard Thaler. 1987, "Further Evidence on Investor Overreaction and Stock Market Seasonality." The Journal of Finance 42(3), 557-81.

Dechow, Patricia, and Richard Sloan, 1997, "Returns to contrarian investment strategies: Tests of naive expectations hypotheses." Journal of Financial Economics 43 (1), 3-27

Dechow, Patricia, Amy Hutton, and Richard Sloan, 2000, "The relation between analysts' forecasts of long-term earnings growth and stock price performance following equity offerings." Contemporary Accounting Research 17(1), 1-32.

DeLong, J. Bradford, Andrei Shleifer, Lawrence Summers, and Robert Waldmann 1990a. "Positive feedback investment strategies and destabilizing rational speculation." Journal of Finance 45(2): 379-95.

DeLong, J. Bradford, Andrei Shleifer, Lawrence Summers, and Robert Waldmann 1990b. "Noise Trader Risk in Financial Markets." Journal of Political Economy 98(4): 703-738.

Dominguez, Kathryn. 1986. "Are Foreign Exchange Forecasts Rational?: New evidence from survey data." Economics Letters 21 (3):277-81.

Easterwood, John, and Stacey Nutt, 1999, "Inefficiency in Analysts' Earnings Forecasts: Systematic Misreaction or Systematic Optimism?” Journal of Finance 54 (5), 1777-1797.

Fama, Eugene and Kenneth French. 1993. "Common Risk Factors in the Returns on Stocks and Bonds." Journal of Financial Economics 33(1): 3 - 56.

Frankel, Jeffrey, and Kenneth Froot. 1987. "Using Survey Data to Test Standard Propositions Regarding Exchange Rate Expectations." American Economic Review 77 (1):133-53.

—. 1988. "Explaining the Demand for Dollars: International Rates of Return, and the Expectations of Chartists and Fundamentalists." In Macroeconomics, agriculture, and the exchange rate. Eds. R. G. Chambers and P. L. Paarlberg. Boulder, CO: Westview Press.

Gennaioli, Nicola, Yueran Ma, and Andrei Shleifer. 2016. "Expectations and Investment." NBER Macroeconomics Annual 30: 379-442.

Gennaioli, Nicola, and Andrei Shleifer. 2010. "What Comes to Mind." Quarterly Journal of Economics 125 (4): 1399-1433.

Glaeser, Edward, and Charles Nathanson. 2015. "Housing Bubbles.” Handbook of Regional and Urban Economics 5: 701-751.

Greenwood, Robin, and Andrei Shleifer. 2014. "Expectations of Returns and Expected Returns." Review of Financial Studies 27 (3): 714-746. 
Hong, Harrison and Jeremy Stein. 1999. "A Unified Theory of Underreaction, Momentum Trading, and Overreaction in Asset Markets.” Journal of Finance 56 (6): 2143 - 2184.

Kahneman, Daniel, and Amos Tversky. 1972. "Subjective Probability: A Judgment of Representativeness." Cognitive Psychology 3 (3): 430-454.

Kahneman, Daniel, and Amos Tversky. 1973. "On the Psychology of Prediction." Psychological Review 80 (4): 237-251.

Lakonishok, Joseph, Andrei Shleifer, and Robert Vishny. 1994. "Contrarian Investment, Extrapolation, and Risk." Journal of Finance 49 (5):1541-78.

La Porta, Rafael. 1996. "Expectations and the Cross-Section of Stock Returns." Journal of Finance 51 (5), 1715-1742.

La Porta, Rafael, Josef Lakonishok, Andrei Shleifer, and Robert Vishny. 1997. "Good News For Value Stocks: Further Evidence On Market Efficiency." Journal of Finance 52(2): 859-874.

Marcet, Albert and Thomas Sargent. 1989. "Convergence of Least Squares Learning Mechanisms in Self-Referential Linear Stochastic Models.” Journal of Economic Theory 48 (2): 337 368.

Michaely, Roni, and Kent Womack. 1999. "Conflict of Interest and the Credibility of Underwriter Analyst Recommendations.” Review of Financial Studies 12 (4), 653-686.

Pastor, Lubos and Pietro Veronesi. 2003. "Stock Valuation and Learning about Profitability." The Journal of Finance 58 (5): 1749-1789.

Pastor, Lubos and Pietro Veronesi. 2005. "Rational IPO Waves." The Journal of Finance 60 (4): 1713-1757.

Pastor, Lubos and Pietro Veronesi. 2009. "Learning in Financial Markets." Annual Review of Financial Economics 1(1): 361-381.

Rabin, Matthew. 2013. "An Approach to Incorporating Psychology into Economics." American Economic Review103 (3): 617-22.

Shiller, Robert. 1981. "The Use of Volatility Measures in Assessing Market Efficiency." Journal of Finance 36(2): 291 - 304.

Sims, Christopher. 2003. "Implications of Rational Inattention." Journal of Monetary Economics 50 (3): 665-690.

Skinner, Douglas and Richard Sloan. 2002. "Earnings Surprises, Growth Expectations, and Stock Returns or Don't Let an Earnings Torpedo Sink Your Portfolio." Review of Accounting Studies 7 (2), 289-312.

Tversky, Amos, and Daniel Kahneman. 1974. "Judgment under Uncertainty: Heuristics and Biases Biases," Science 185 (4157), 1124-1131.

Tversky, Amos, and Daniel Kahneman. 1983. "Extensional versus Intuitive Reasoning: the Conjunction Fallacy in Probability Judgment." Psychological Review 90 (4): 293-315. 


\section{Appendix.}

\section{A. Proofs}

Proposition 1. Upon observing $g_{i, t} \equiv x_{i, t}-b x_{i, t-1}$, the analyst's believed distribution of firm fundamentals is given by:

$$
h^{\theta}\left(f, g_{i, t}\right)=h\left(f, g_{i, t}\right) \cdot\left[R\left(f, g_{i, t}\right)\right]^{\theta} \cdot Z
$$

where $Z^{-1}=\int h\left(f, g_{i, t}\right) \cdot\left[R\left(f, g_{i, t}\right)\right]^{\theta} \cdot d f$ and

$$
\operatorname{Rep}\left(f, g_{i, t}\right)=\exp \left\{\frac{\left(\hat{f}_{i, t}-a \hat{f}_{i, t-1}\right)\left(2 f-a \hat{f}_{i, t-1}-\hat{f}_{i, t}\right)}{2 \sigma_{f}^{2}}\right\}
$$

We expand the above expression using the assumption that $h\left(f, g_{i, t}\right)$ is normally distributed with variance $\sigma_{f}^{2}$ and mean:

$$
\hat{f}_{i, t}=a \hat{f}_{i, t-1}+K\left(g_{i, t}-a \hat{f}_{i, t-1}\right)
$$

We find:

$$
h^{\theta}\left(f, g_{i, t}\right)=Z \cdot \exp \frac{1}{2 \sigma_{f}^{2}}\left\{-\left(f-\hat{f}_{i, t}\right)^{2}+\theta\left(\hat{f}_{i, t}-a \hat{f}_{i, t-1}\right)\left(2 f-a \hat{f}_{i, t-1}-\hat{f}_{i, t}\right)\right\}
$$

The exponent then reads:

$$
\begin{aligned}
-\left(f-\hat{f}_{i, t}\right)^{2}+ & \theta\left(\hat{f}_{i, t}-a \hat{f}_{i, t-1}\right)\left(2 f-a \hat{f}_{i, t-1}-\hat{f}_{i, t}\right) \\
& =-\left(f-\left(\hat{f}_{i, t}+\theta\left(\hat{f}_{i, t}-a \hat{f}_{i, t-1}\right)\right)\right)^{2}+c\left(\hat{f}_{i, t}, \hat{f}_{i, t-1}\right)
\end{aligned}
$$


where $c\left(\hat{f}_{i, t}, \hat{f}_{i, t-1}\right)$ is a constant (does not depend on $\left.f\right)$. Taking normalization into account, we find

$$
h^{\theta}\left(f, g_{i, t}\right)=\frac{1}{\sqrt{2 \pi \sigma_{f}^{2}}} e^{-\frac{\left(f-\left(\hat{f}_{i, t}+\theta\left(\hat{f}_{i, t}-a \hat{f}_{i, t-1}\right)\right)\right)^{2}}{2 \sigma_{f}^{2}}}
$$

Using Equation (3) for the Bayesian expectation $\hat{f}_{i, t}$, the mean of this distribution can be written:

$$
\hat{f}_{i, t}^{\theta}=\hat{f}_{i, t}+\theta\left(\hat{f}_{i, t}-a \hat{f}_{i, t-1}\right)=a \hat{f}_{i, t-1}+K(1+\theta)\left(g_{i, t}-a \hat{f}_{i, t-1}\right)
$$

Proposition 2. Denote by $\lambda_{H}>0$ the threshold in expected growth rate above which a firm is classified as HLTG (i.e., it is in the top decile). From the definition of LTG in Section V, firm $i$ is classified as HLTG at time $t$ provided:

$$
L T G_{i, t}=-\varphi_{h} x_{i, t}+\vartheta_{h} \hat{f}_{i, t}^{\theta} \geq \lambda_{H}
$$

where we have defined $\varphi_{h} \equiv\left(1-b^{h}\right)$ and $\vartheta_{h} \equiv a^{h} \frac{1-(b / a)^{h}}{1-(b / a)}$. This can be written as:

$$
-\varphi_{h} x_{i, t}+\vartheta_{h} a\left(1-K^{\prime}\right) \hat{f}_{i, t-1}+\vartheta_{h} K^{\prime}(1-b) x_{i, t-1}+\vartheta_{h} K^{\prime}\left(x_{i, t}-x_{i, t-1}\right) \geq \lambda_{H},
$$

where $K^{\prime} \equiv K(1+\theta)$. The left hand side of the above condition is a linear combination of mean zero normally distributed random variables. Denote it by $L H S_{i, t}$. By linear regression, the average growth rate $x_{i, t}-x_{i, t-1}$ experienced by firms whose $L H S_{i, t}$ is equal to $\lambda$ is given by:

$$
\mathbb{E}\left[x_{i, t}-x_{i, t-1} \mid L H S_{i, t}=\lambda\right]=\frac{\operatorname{cov}\left(x_{i, t}-x_{i, t-1}, L H S_{i, t}\right)}{\operatorname{var}\left(L H S_{i, t}\right)} \lambda
$$


Because for HLTG firms $\lambda \geq \lambda_{H}>0$, their pre-formation growth is positive, $\mathbb{E}\left[x_{i, t}-\right.$ $\left.x_{i, t-1} \mid L H S_{i, t}=\lambda\right]>0$, provided $\operatorname{cov}\left(x_{i, t}-x_{i, t-1}, L H S_{i, t}\right)>0$. This occurs when the expression:

$$
\left[K^{\prime} \vartheta_{h}(1+b)-\varphi_{h}\right]\left(\operatorname{var}\left(x_{i, t}\right)-\operatorname{cov}\left(x_{i, t}, x_{i, t-1}\right)\right)+\vartheta_{h} a\left(1-K^{\prime}\right) \operatorname{cov}\left(x_{i, t}-x_{i, t-1}, \hat{f}_{i, t-1}\right)
$$

is positive. For convenience, rewrite this as:

$$
A\left[\operatorname{var}\left(x_{i, t}\right)-\operatorname{cov}\left(x_{i, t}, x_{i, t-1}\right)\right]+B \operatorname{cov}\left(x_{i, t}-x_{i, t-1}, \hat{f}_{i, t-1}\right)
$$

It is useful to rewrite the first term as:

$$
A\left[(1-b) \operatorname{var}\left(x_{i, t}\right)-a \cdot \operatorname{cov}\left(f_{i, t-1}, x_{i, t-1}\right)\right]=A\left[\frac{\operatorname{var}(\epsilon)}{1+b}+\frac{\operatorname{var}\left(f_{i, t}\right)}{1-a b} \frac{1-a}{1+b}\right]
$$

where we used $\operatorname{var}\left(x_{i, t}\right)=\frac{1}{1-b^{2}}\left[\operatorname{var}(\epsilon)+\frac{1+a b}{1-a b} \operatorname{var}\left(f_{i, t}\right)\right]$. The second term reads:

$$
\begin{aligned}
\operatorname{cov}\left(x_{i, t}-x_{i, t-1}, a(1-K) \hat{f}_{i, t-2}+K\left(f_{i, t-1}+\epsilon_{i, t-1}\right)\right) \\
=a(1-K) \operatorname{cov}\left(x_{i, t}-x_{i, t-1}, \hat{f}_{i, t-2}\right)-(1-b) K \operatorname{cov}\left(x_{i, t-1}, f_{i, t-1}\right) \\
-(1-b) K \operatorname{var}(\epsilon)+\operatorname{aKvar}\left(f_{i, t}\right)
\end{aligned}
$$

We can show that:

$$
\operatorname{cov}\left(x_{i, t}-x_{i, t-1}, \hat{f}_{i, t-2}\right)>a^{2} \operatorname{cov}\left(f_{i, t}, \hat{f}_{i, t-2}\right)-b(1-b) \operatorname{cov}\left(x_{i, t-2}, f_{i, t-2}\right)-a(1-b) \operatorname{var}\left(f_{i, t}\right)
$$

where we used $\operatorname{cov}\left(x_{i, t-1}, f_{i, t-2}\right)>\operatorname{cov}\left(x_{i, t-1}, \hat{f}_{i, t-2}\right)$. Thus:

$$
\begin{aligned}
\operatorname{cov}\left(x_{i, t}-x_{i, t-1}, a(1-K) \hat{f}_{i, t-2}+K\left(f_{i, t-1}+\epsilon_{i, t-1}\right)\right) & \\
> & \operatorname{var}\left(f_{i, t}\right)\left[\frac{a K}{1-a^{2}(1-K)}-(1-b)\left(K+a^{2}(1-K)+\frac{a b}{1-a b}\right)\right] \\
& -(1-b) K \operatorname{var}(\epsilon)
\end{aligned}
$$


So putting the two terms together we find

$$
\begin{aligned}
\operatorname{var}(\epsilon)\left[\frac{A}{1+b}\right. & -(1-b) K B] \\
& +\operatorname{var}\left(f_{i, t}\right)\left[\frac{A}{1+b} \frac{1-a}{1-a b}\right. \\
& \left.+B\left[\frac{a K}{1-a^{2}(1-K)}-(1-b)\left(K+a^{2}(1-K)+\frac{a b}{1-a b}\right)\right]\right]
\end{aligned}
$$

A sufficient condition that makes both terms positive is:

$$
b^{h}+\vartheta_{h}\left[\frac{K^{\prime}-a}{1-a}\right]>1
$$

This condition is easier to satisfy for low mean reversion (large $b$ ), large signal to noise ratio (large $\mathrm{K}$ ) and for strong overreaction (large $\theta$ ). It is trivially satisfied when $b=1$ provided $K(1+\theta) \geq a$ (which holds in our calibration).

Proposition 3. From the law of motion of earnings we have that:

$$
\mathbb{E}\left(x_{i, t+h}-x_{i, t} \mid H L T G_{t}\right)=\mathbb{E}\left(-\varphi_{h} x_{i, t}+\vartheta_{h} f_{i, t} \mid H L T G_{t}\right)
$$

Because rational estimation errors $u_{i, t} \equiv f_{i, t}-\hat{f}_{i, t}$ are on average zero, we also have that:

$$
\mathbb{E}\left(x_{i, t+h}-x_{i, t} \mid H L T G_{t}\right)=\mathbb{E}\left(-\varphi_{h} x_{i, t}+\vartheta_{h} \hat{f}_{i, t} \mid H L T G_{t}\right) .
$$

This implies that the average forecast error entailed in LTG is equal to:

$$
\begin{gathered}
\mathbb{E}\left(x_{i, t+h}-x_{i, t}-L T G_{i, t} \mid H L T G_{t}\right)=\vartheta_{h} \mathbb{E}\left(\hat{f}_{i, t}-\hat{f}_{i, t}^{\theta} \mid H L T G_{t}\right)= \\
-\vartheta_{h} K \theta \mathbb{E}\left(x_{i, t}-b x_{i, t-1}-a \hat{f}_{i, t-1} \mid H L T G_{t}\right)=-\vartheta_{h} K \theta \mathbb{E}\left(\eta_{i, t}+\epsilon_{i, t} \mid H L T G_{t}\right) .
\end{gathered}
$$


The expectation $\mathbb{E}\left(\eta_{i, t}+\epsilon_{i, t} \mid H L T G_{t}\right)$ is positive because HLTG firms have positive recent performance (see Lemma 1). Under rationality, $\theta=0$, forecast errors are unpredictable. Under diagnostic expectations, $\theta>0$, forecast errors are predictably negative for the HLTG group. Conversely, the same argument shows that they are predictably positive for the LLTG group.

Proposition 4. The average LTG at future date $t+s, s \geq 1$, in the HLTG group is equal to:

$$
\begin{gathered}
\mathbb{E}\left(L T G_{i, t+s} \mid H L T G_{t}\right)=\mathbb{E}\left(-\varphi_{h} x_{i, t+s}+\vartheta_{h} \hat{f}_{i, t+s}^{\theta} \mid H L T G_{t}\right)= \\
\mathbb{E}\left(-\varphi_{h} x_{i, t+s}+\vartheta_{h} \hat{f}_{i, t+s}+\vartheta_{h}\left(\hat{f}_{i, t+s}^{\theta}-\hat{f}_{i, t+s}\right) \mid H L T G_{t}\right)= \\
\mathbb{E}\left(-\varphi_{h} x_{i, t+s}+\vartheta_{h} \hat{f}_{i, t+s}+\vartheta_{h} K \theta\left(g_{i, t+s}-a \hat{f}_{i, t+s-1}\right) \mid H L T G_{t}\right)= \\
-\varphi_{h} b^{s} x_{i, t}+\vartheta_{h} a^{s} \hat{f}_{i, t}
\end{gathered}
$$

where the last equality follows from the fact that within the HLTG group of stocks, $g_{i, t+s}-$ $a \hat{f}_{i, t+s-1}$ is on average zero. This implies that within HLTG stocks future $L T G_{i, t+s}$ on average mean reverts, as implied by the power terms $b^{s}$ and $a^{s}$. This occurs regardless of whether expectations are rational or diagnostic because $-\varphi_{h} b^{s} x_{i, t}+\vartheta_{h} a^{s} \hat{f}_{i, t}$ does not depend on $\theta$. Between the formation date $t$ and $t+1$, however, mean reversion is stronger under diagnostic expectations. In fact, the condition

$$
\mathbb{E}\left(g_{i, t+s}-a \hat{f}_{i, t+s-1} \mid H L T G_{t}\right)>0
$$

holds if and only if

$$
b^{h}+\vartheta_{h} K^{\prime}>1
$$


This condition is implied by the assumption of Proposition 2 (Equation 7), provided $K^{\prime}>1$, which holds in the calibration. We then find:

$$
\mathbb{E}\left(L T G_{i, t+1} \mid H L T G_{t}\right)-\mathbb{E}\left(L T G_{i, t} \mid H L T G_{t}\right)=\mathbb{E}\left(L T G_{i, t+1}^{\theta=0} \mid H L T G_{t}\right)-\mathbb{E}\left(L T G_{i, t}^{\theta=0} \mid H L T G_{t}\right)-\Psi \theta
$$

where $\Psi=\vartheta_{h} K \mathbb{E}\left(g_{i, t+s}-a \hat{f}_{i, t+s-1}\right)>0$ (and $\theta>0$ ). This is because under diagnostic expectation the average $L T G_{i, t}$ in the HLTG group is inflated relative to the rational benchmark. The converse holds for stocks in the LLTG group at time $t$.

Proposition 5. The realized return at time $t$ on HLTG stocks is equal to the average:

$$
\mathbb{E}\left(\frac{P_{i, t}+D_{i t}}{P_{i, t-1}} \mid H L T G_{t}\right)=R+\mathbb{E}\left(\frac{P_{i, t}-\mathbb{E}_{i, t-1}^{\theta}\left(P_{i t}\right)+D_{i, t}-\mathbb{E}_{t-1}^{\theta}\left(D_{i, t}\right)}{P_{i, t-1}} \mid H L T G_{t}\right)
$$

An individual stock $i$ in the HLTG portfolio therefore experiences positive abnormal returns pre formation provided:

$$
P_{i, t}-\mathbb{E}_{t-1}^{\theta}\left(P_{i, t}\right)+D_{i, t}-\mathbb{E}_{t-1}^{\theta}\left(D_{i, t}\right)>0
$$

Consider the first term $P_{i, t}-\mathbb{E}_{t-1}^{\theta}\left(P_{i, t}\right)$. Because prices are equal to discounted future dividends:

$$
P_{i, t}-\mathbb{E}_{t-1}^{\theta}\left(P_{i, t}\right)=\sum_{s \geq 1} \frac{\mathbb{E}_{t}^{\theta}\left(D_{i, t+s}\right)-\mathbb{E}_{t-1}^{\theta}\left(D_{i, t+s}\right)}{R^{s}}
$$

which implies that abnormal returns are induced by an upward revision $\mathbb{E}_{t}^{\theta}\left(D_{i, t+s}\right)$ $\mathbb{E}_{t-1}^{\theta}\left(D_{i, t+s}\right)>0$ of investors' beliefs of future dividends. Using previous notation, we have that:

$$
\mathbb{E}_{t}^{\theta}\left(D_{i, t+s}\right)=\mathbb{E}_{t}^{\theta}\left(e^{x_{i, t+s}}\right)=e^{b^{s} x_{i, t}+\vartheta_{s} \hat{f}_{i, t}^{\theta}+\frac{1}{2} \operatorname{Var}_{t}\left(x_{t+s}\right)}
$$


As a result, we have that $\mathbb{E}_{t}^{\theta}\left(D_{i, t+s}\right)-\mathbb{E}_{t-1}^{\theta}\left(D_{i, t+s}\right)>0$ provided that, on average in HLTG:

$$
b^{s} x_{i, t}+\vartheta_{s} \hat{f}_{i, t}^{\theta}>b^{s} \mathbb{E}_{t-1}^{\theta}\left(x_{i, t}\right)+\vartheta_{s} \mathbb{E}_{t-1}^{\theta}\left(f_{i, t}\right)
$$

We have:

$$
\begin{aligned}
b^{s}\left(x_{i, t}-\mathbb{E}_{t-1}^{\theta}\left(x_{i, t}\right)\right)+\vartheta_{s}\left(\hat{f}_{i, t}^{\theta}-\mathbb{E}_{t-1}^{\theta}\left(\hat{f}_{i, t}^{\theta}\right)\right) \\
=b^{s}\left(S_{i, t}-a K \theta S_{i, t-1}\right)+\vartheta_{s}\left(K(1+\theta) S_{i, t}-a K \theta S_{i, t-1}\right) \\
=S_{i, t}\left(b^{s}+\vartheta_{s} K\right)+\theta K\left(\vartheta_{s} S_{i, t}-\vartheta_{s+1} S_{i, t-1}\right)
\end{aligned}
$$

where $S_{t}=x_{i, t}-b x_{i, t-1}-a \hat{f}_{i, t-1}$ is the news, or surprise, at time $t$. Intuitively, this suggests that high returns at $t$ are associated with surprises $S_{i, t}$ that are not only positive, but also (for $\theta>$ 0) sufficiently large compared to surprises in the previous period. Rewriting the above as $A S_{i, t}-$ $B A S_{i, t-1}$ with $A=b^{s}+\vartheta_{s} K(1+\theta)$ and $B=\theta K \vartheta_{s+1}$, we then have:

$$
\mathbb{E}\left[A S_{i, t}-B A S_{i, t-1} \mid L T G_{i, t}=\lambda\right]=\frac{\operatorname{cov}\left(A S_{i, t}-B A S_{i, t-1}, L T G_{i, t}\right)}{\operatorname{var}\left(L T G_{i, t}\right)} \lambda
$$

We can write $L T G_{i, t}$ as:

$$
L T G_{i, t}=\left[\vartheta_{s} K(1+\theta)-\varphi_{s}\right] S_{i, t}-\left[\varphi_{s}(b+a K)-a \vartheta_{s} K\right] S_{i, t-1}+\text { terms in } t-2
$$

Because surprises in period $t$ are uncorrelated with information at different periods, the numerator of the expectation above then reads:

$$
\begin{aligned}
\operatorname{cov}\left(A S_{i, t}-B\right. & \left.A S_{i, t-1}, L T G_{i, t}\right) \\
& =A\left[\vartheta_{s} K(1+\theta)-\varphi_{s}\right] \operatorname{var}\left(S_{i, t}\right)-B\left[\varphi_{s}(b+a K)-\operatorname{a\vartheta _{s}} K\right] \operatorname{var}\left(S_{i, t-1}\right) \\
& =\operatorname{var}\left(S_{i, t}\right)\left[A\left[\vartheta_{s} K(1+\theta)-\varphi_{s}\right]-B\left[\varphi_{s}(b+a K)-a \vartheta_{s} K\right]\right]
\end{aligned}
$$

This is positive provided 


$$
\vartheta_{s} K(1+\theta)+b^{s}>1+2 \theta K \vartheta_{s+1}\left[\varphi_{s}(b+a K)-a \vartheta_{s} K\right]
$$

(which holds in our calibration). The assumption of Proposition 2 guarantees $\vartheta_{s} K(1+\theta)+b^{s}>$ 1. Thus, under rational expectations, $\theta=0$, the condition holds trivially. For $\theta>0$, a sufficient condition for the above to hold is that $\vartheta_{s} K>\varphi_{s}\left(\frac{b}{a}+K\right)$. Under this condition, $\mathbb{E}\left[x_{i, t}+L T G_{i, t}-\right.$ $\left.x_{i, t-1}-L T G_{i, t-1} \mid L T G_{i, t} \geq \lambda_{h}\right]$ is positive, and the result follows. Note that this is implied by the assumption of Proposition 2 provided $\frac{b}{a}+K<\frac{1}{1+\theta}$.

Proposition 6. As shown in Equation (9), a stock's average return going forward into the next period is equal to:

$$
\frac{\mathbb{E}_{t}\left(P_{i, t+1}+D_{i, t+1}\right)}{\mathbb{E}_{t}^{\theta}\left(P_{i, t+1}+D_{i, t+1}\right)} R
$$

Note that $D_{i, t+s}=e^{x_{i, t+s}}$ and that, given that price is the discounted sum of future dividends:

$$
P_{i, t+1}+D_{i, t+1}=\sum_{s \geq 0} \frac{D_{i, t+1+s}}{R^{s}}=\sum_{s \geq 0} e^{x_{i, t+1+s}-s \ln R},
$$

where, as usual, we assume that $\ln R$ is large enough that the sum converges. Given lognormality, we have that:

$$
\mathbb{E}_{t}^{\theta}\left(P_{i, t+1}+D_{i, t+1}\right)=\sum_{s \geq 0} e^{\mathbb{E}_{t}^{\theta}\left(x_{i, t+1+s}\right)-\operatorname{sln} R+\frac{1}{2} \operatorname{Var}_{t}\left(x_{i, t+1+s}\right)},
$$

where rational expectations correspond to the special case of $\theta=0$. For $\theta=0$, then, the numerator and the denominator of Equation (9) are equal, so that the average realized return is 
equal to the realized return $R$ for all firms. As a result, the average realized post-formation return of the HLTG and LLTG portfolios should be equal to the required return $R$.

To see the role of diagnostic expectations, note that $\theta$ only influences the expected log dividend $\mathbb{E}_{t}^{\theta}\left(x_{i, t+1+s}\right)$, but not the perceived variance $\operatorname{Var}_{t}\left(x_{i, t+1+s}\right)$. In particular:

$$
\mathbb{E}_{t}^{\theta}\left(x_{i, t+s+1}\right)=b^{s+1} x_{t}+a^{s+1} \frac{1-(b / a)^{s+1}}{1-(b / a)}\left[\hat{f}_{i, t}+K \theta\left(g_{i, t}-a \hat{f}_{i, t-1}\right)\right]
$$

This implies that:

$$
\begin{aligned}
& \frac{\partial \mathbb{E}_{t}^{\theta}\left(P_{i, t+1}+D_{i, t+1}\right)}{\partial \theta} \\
& \quad=K \theta\left(g_{i, t}-a \hat{f}_{i, t-1}\right) \sum_{s \geq 0} a^{s+1} \frac{1-(b / a)^{s+1}}{1-(b / a)} e^{\mathbb{E}_{t}^{\theta}\left(x_{i, t+1+s}\right)-\sin R+\frac{1}{2} \operatorname{Var}_{t}\left(x_{i, t+1+s}\right)}
\end{aligned}
$$

Under diagnostic expectations, pre-formation news drive mispricing. HLTG stocks experience positive surprises before formation, namely $\left(g_{i, t}-a \hat{f}_{i, t-1}\right)>0$. As a result, the diagnostic expectation $\mathbb{E}_{t}^{\theta}\left(P_{i, t+1}+D_{i, t+1}\right)$ is above the rational counterpart, so that realized post formation returns are on average below the required return $R$. For LLTG the opposite is true.

Proposition 7. Regressing $x_{i, t+h}-x_{i, t}-L T G_{i, t}$ on $L T G_{i, t}-L T G_{i, t-k}$ yields a coefficient

$$
\beta=\frac{\operatorname{cov}\left(x_{i, t+h}-x_{i, t}-L T G_{i, t} L T G_{i, t}-L T G_{i, t-k}\right)}{\operatorname{var}\left(L T G_{i, t}-L T G_{i, t-k}\right)}
$$

The forecast error in the denominator reads

$$
x_{i, t+h}-x_{i, t}-\mathbb{E}_{t}\left(x_{i, t+h}-x_{i, t}\right)-\theta \vartheta_{h} K\left(x_{i, t}-b x_{i, t-1}-a \hat{f}_{i, t-1}\right)
$$


The first two terms include only shocks after $t$ and do not co-vary with any quantity at $t$. The last term arises only for $\theta>0$, and captures the overreaction to news at $t$ embedded in $L T G_{i, t}$. We thus have

$$
\beta \propto-\theta \cdot \operatorname{cov}\left(x_{i, t}-b x_{i, t-1}-a \hat{f}_{i, t-1}, L T G_{i, t}-L T G_{i, t-k}\right)
$$

Intuitively, a positive covariance means that positive surprises at $t$ tend to be associated with upward revisions in LTG. The second argument reads:

$$
\begin{gathered}
-\varphi_{h}\left(x_{i, t}-x_{i, t-1}\right)+\vartheta_{h} a\left(1-K^{\prime}\right)\left(\hat{f}_{i, t-1}-\hat{f}_{i, t-2}\right)+\vartheta_{h} K^{\prime}\left(x_{i, t}-b x_{i, t-1}-a \hat{f}_{i, t-1}\right) \\
-\vartheta_{h} K^{\prime}\left(x_{i, t-1}-b x_{i, t-2}-a \hat{f}_{i, t-2}\right)
\end{gathered}
$$

The surprise at $t$ does not covary with either the update in beliefs at $t-1$ (second term), nor with the surprise at $t-1$ (last term), so these drop out. Write the first term as

$$
-\varphi_{h}\left(x_{i, t}-x_{i, t-1}\right)=-\varphi_{h}\left(x_{i, t}-b x_{i, t-1}-a \hat{f}_{i, t-1}\right)-\varphi_{h}\left((1-b) x_{i, t-1}-a \hat{f}_{i, t-1}\right)
$$

Again, because surprises at $t$ are not predictable from information at $t-1$, the second term drops out. We therefore get $\left(\vartheta_{h} K^{\prime}-\varphi_{h}\right) \operatorname{var}\left(x_{i, t}-b x_{i, t-1}-a \hat{f}_{i, t-1}\right)$ which is positive if and only if

$$
b^{h}+\vartheta_{h} K(1+\theta)>1
$$

This condition is weaker than that of Proposition 2 provided $K(1+\theta)>1$, which holds in our calibration.

\section{B. Robustness of LLTG-HLTG Return Differential}



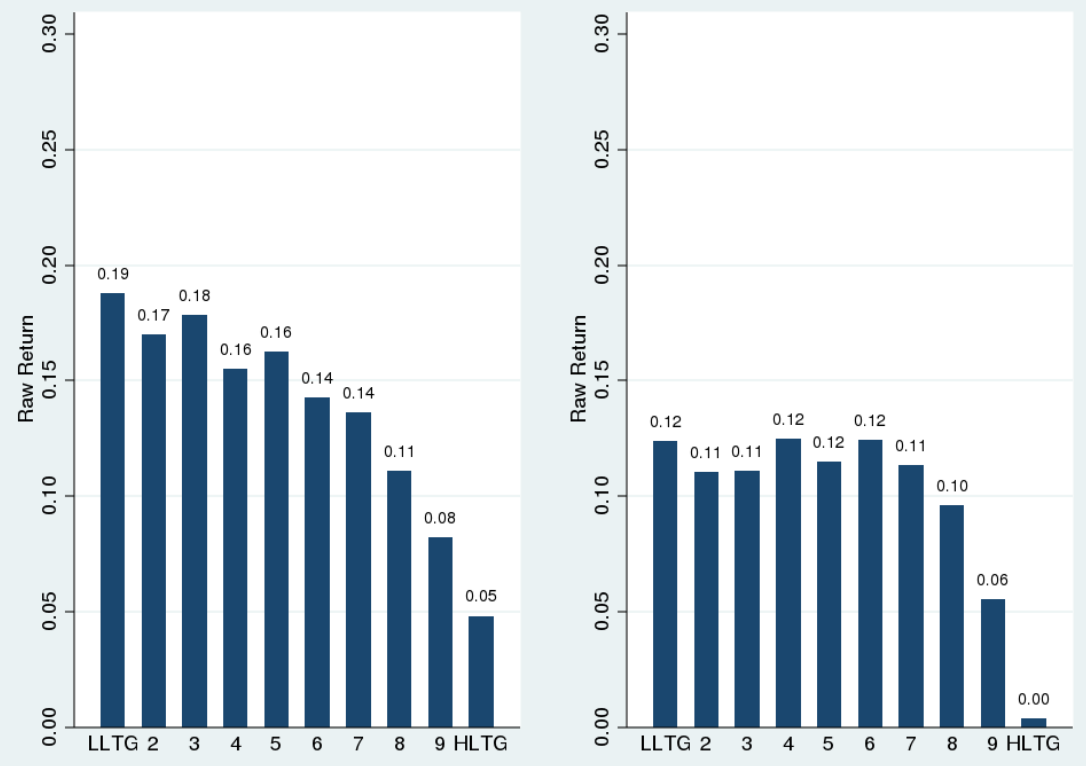

Figure B.1. Annual Returns for Portfolios Formed on LTG. In December of each year between 1981 and 2015, we form decile portfolios based on ranked analysts' expected growth in earnings per share. The graph on the left (right) shows the geometric average one-year equally-weighted return over the subsequent calendar year during the period 1982-1997 (1998-2015).

\section{Table B.1 - Average Beta is increasing across LTG Portfolios}

We estimate slope coefficients from the following OLS regression

$$
r e t_{i, t}-r f_{t}=\alpha_{i}+\beta_{i} *\left(r e t_{i, t}-r f_{i, t}\right)+\varepsilon_{i, t}
$$

where ret ${ }_{i, t}$ is the monthly return for firm $i, \mathrm{rf}_{t}$ is the risk-free rate (from Ken French's website) in period $t$, $\mathrm{rm}_{t}$ is the return on the equally-weighted index in period $t$ (also from Ken French's website). We estimate the regression using a rolling window of 60 months. The table below reports average $\beta$ s for LTG portfolios.

LTG decile

\begin{tabular}{|c|c|c|c|c|c|c|c|c|c|c|c|}
\hline & 1 & 2 & 3 & 4 & 5 & 6 & 7 & 8 & 9 & 10 & $1-10$ \\
\hline$\beta$ & 0.80 & 0.93 & 1.00 & 1.06 & 1.12 & 1.18 & 1.29 & 1.37 & 1.46 & 1.50 & -0.71 \\
\hline
\end{tabular}

Table B.2 - Annual Returns for Portfolios Formed on LTG and six-month momentum 
In December of each year between 1981 and 2015, we independently form ten portfolios based on ranked analysts' expected growth in earnings per share and three portfolios (i.e. bottom $30 \%$, middle $40 \%$, and top $30 \%$ ) based on sixmonth momentum (i.e. July-December of year t). The table below reports the average one-year return over the subsequent calendar year for equally-weighted portfolios.

\begin{tabular}{rcccc}
\multicolumn{5}{c}{ Rank of Returns in the Previous 6 Months } \\
\hline LTG & Bottom 30\% & Middle & Top 30\% & $\begin{array}{c}\text { Top- } \\
\text { Bottom }\end{array}$ \\
\hline 1 & $9.8 \%$ & $15.5 \%$ & $17.7 \%$ & $7.8 \%$ \\
2 & $9.2 \%$ & $15.3 \%$ & $14.0 \%$ & $5 \%$ \\
3 & $9.8 \%$ & $15.0 \%$ & $15.4 \%$ & $6 \%$ \\
4 & $9.7 \%$ & $14.0 \%$ & $13.3 \%$ & $4 \%$ \\
5 & $8.9 \%$ & $14.9 \%$ & $14.7 \%$ & $6 \%$ \\
6 & $10.3 \%$ & $14.4 \%$ & $14.3 \%$ & $4 \%$ \\
7 & $7.1 \%$ & $12.5 \%$ & $15.5 \%$ & $8 \%$ \\
8 & $5.6 \%$ & $11.2 \%$ & $13.0 \%$ & $7 \%$ \\
9 & $5.8 \%$ & $8.0 \%$ & $8.6 \%$ & $3 \%$ \\
10 & $-1.0 \%$ & $4.6 \%$ & $8.2 \%$ & $9 \%$ \\
\hline $1-10$ & $10.9 \%$ & $10.9 \%$ & $9.4 \%$ & $-1.4 \%$
\end{tabular}




\section{Table B.3 - Factor Regressions}

In December of each year between 1981 and 2015, we form decile portfolios based on ranked analysts' expected growth in earnings per share and compute the equally-weighted monthly return for decile portfolios. Panel A reports the results of regressing the equally-weighted monthly returns on the market factor. Panel B adds the small-minusbig factor and the high-minus-low book-to-market factor. Panel $\mathrm{C}$ adds the robust-minus-weak operating profitability factor and the conservative-minus-aggressive investment factor. All data on factor returns is Ken French's website.

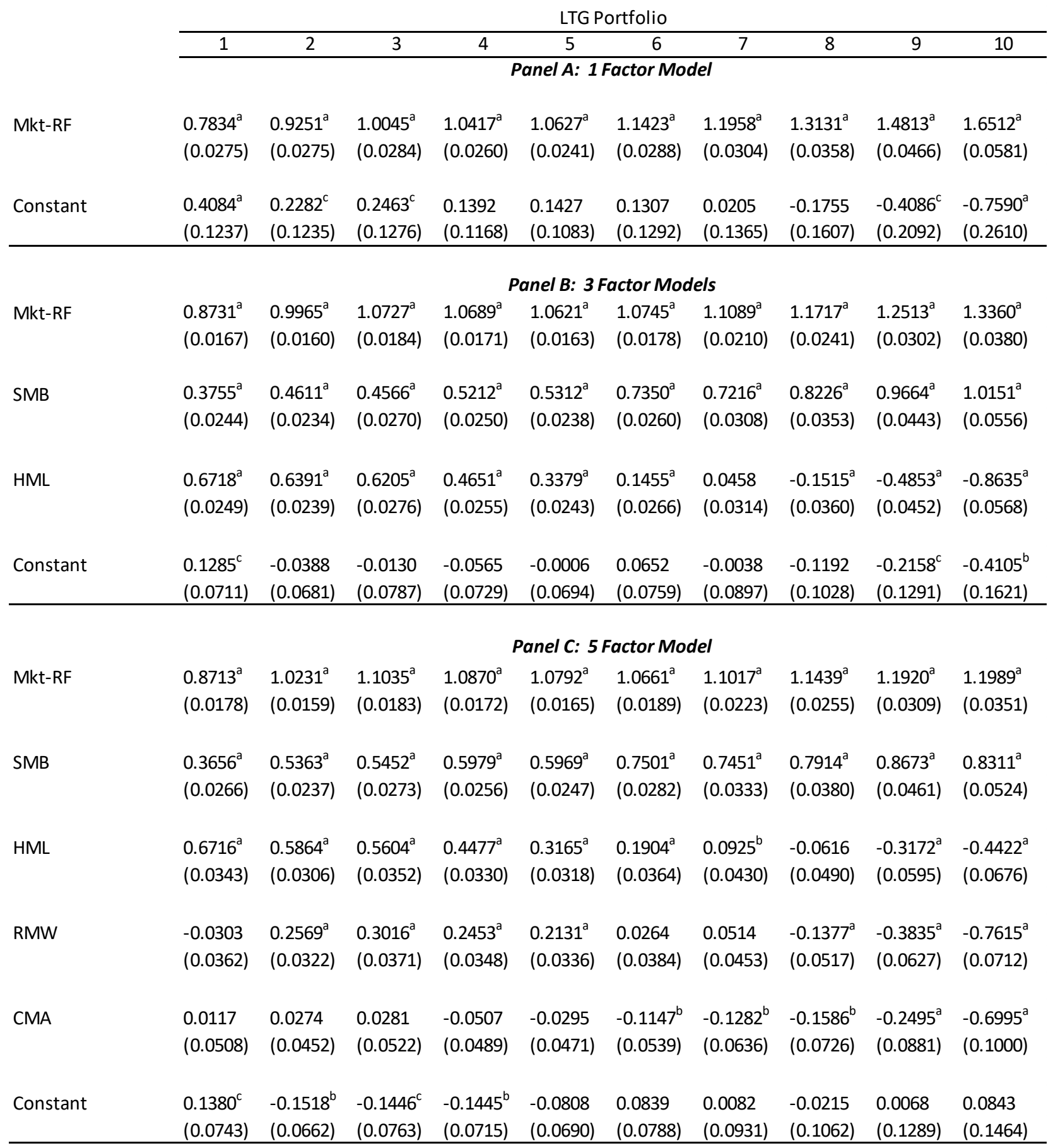




\section{Kernel of Truth}

\section{C.1: EPS growth as a measure of performance}
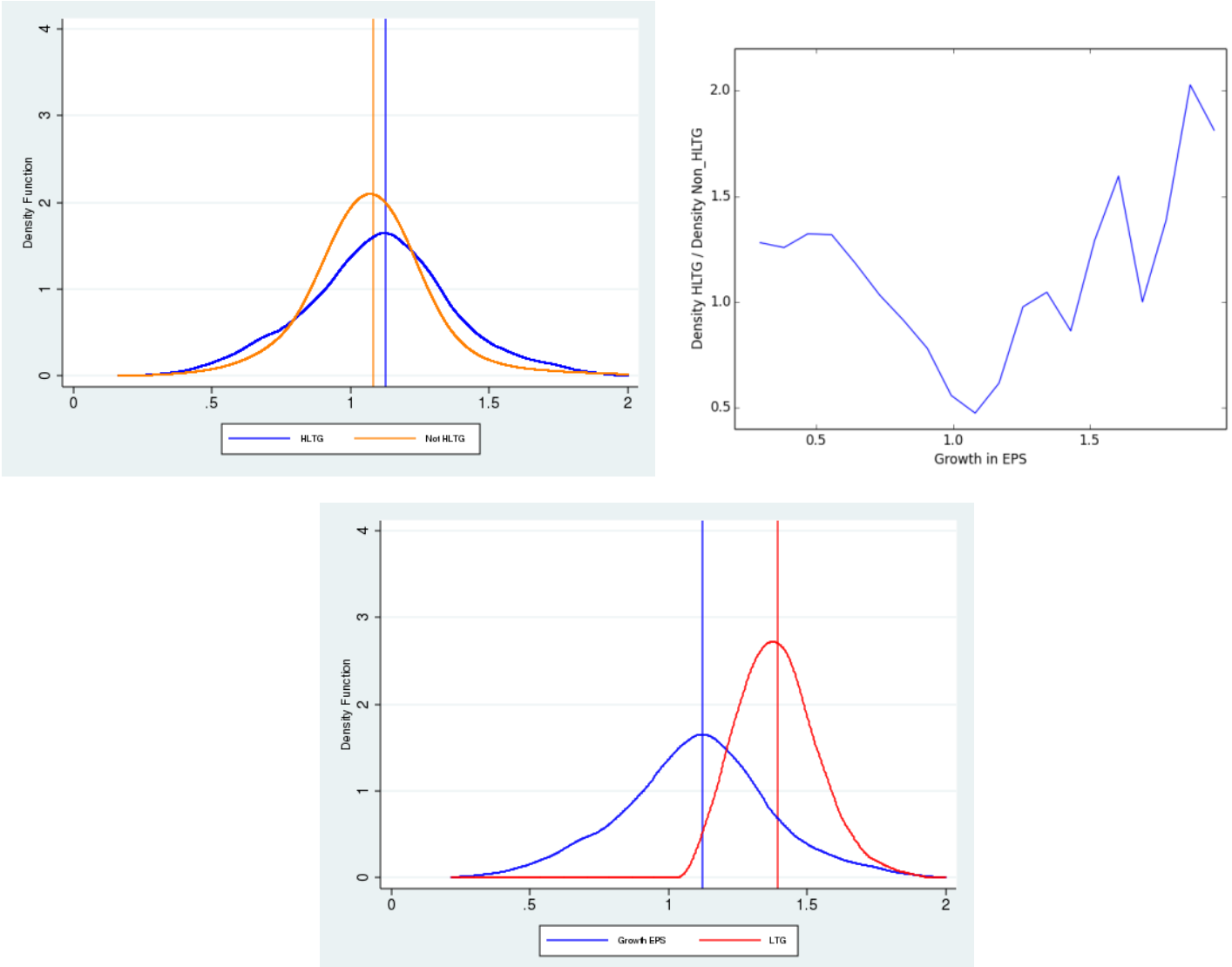

Figure C.1. Long term annualized EPS growth for the HLGT portfolio (top left panel). Representativeness of EPS growth for HLTG (top right panel). EPS growth and LTG forecasts for HLTG (bottom panel).
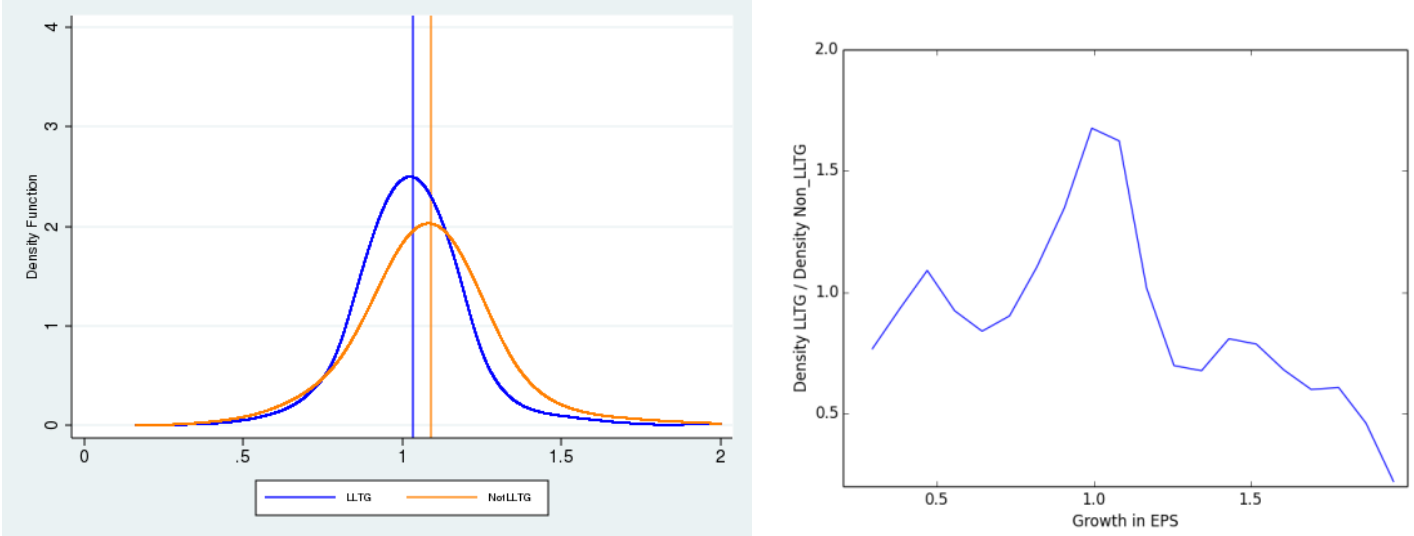


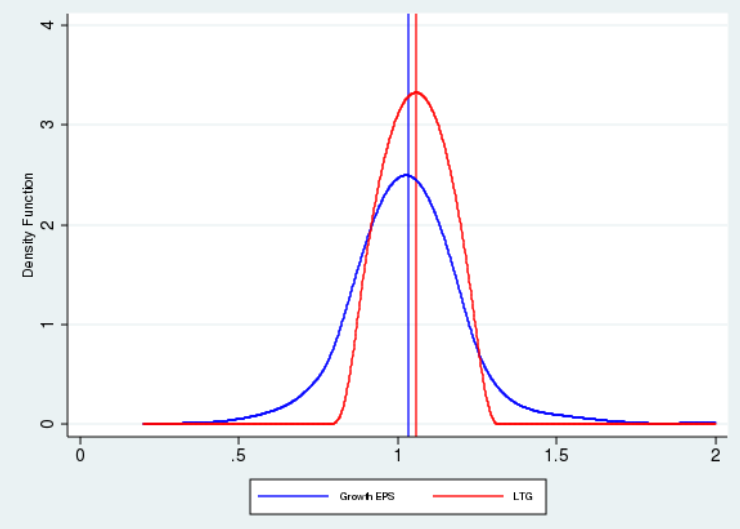

Figure C.2. Long term annualized EPS growth for the LLGT portfolio (top left panel). Representativeness of EPS growth for LLTG (top right panel). EPS growth and LTG forecasts for LLTG (bottom panel).

\section{C.2: Robustness: RMC growth as a measure of performance}
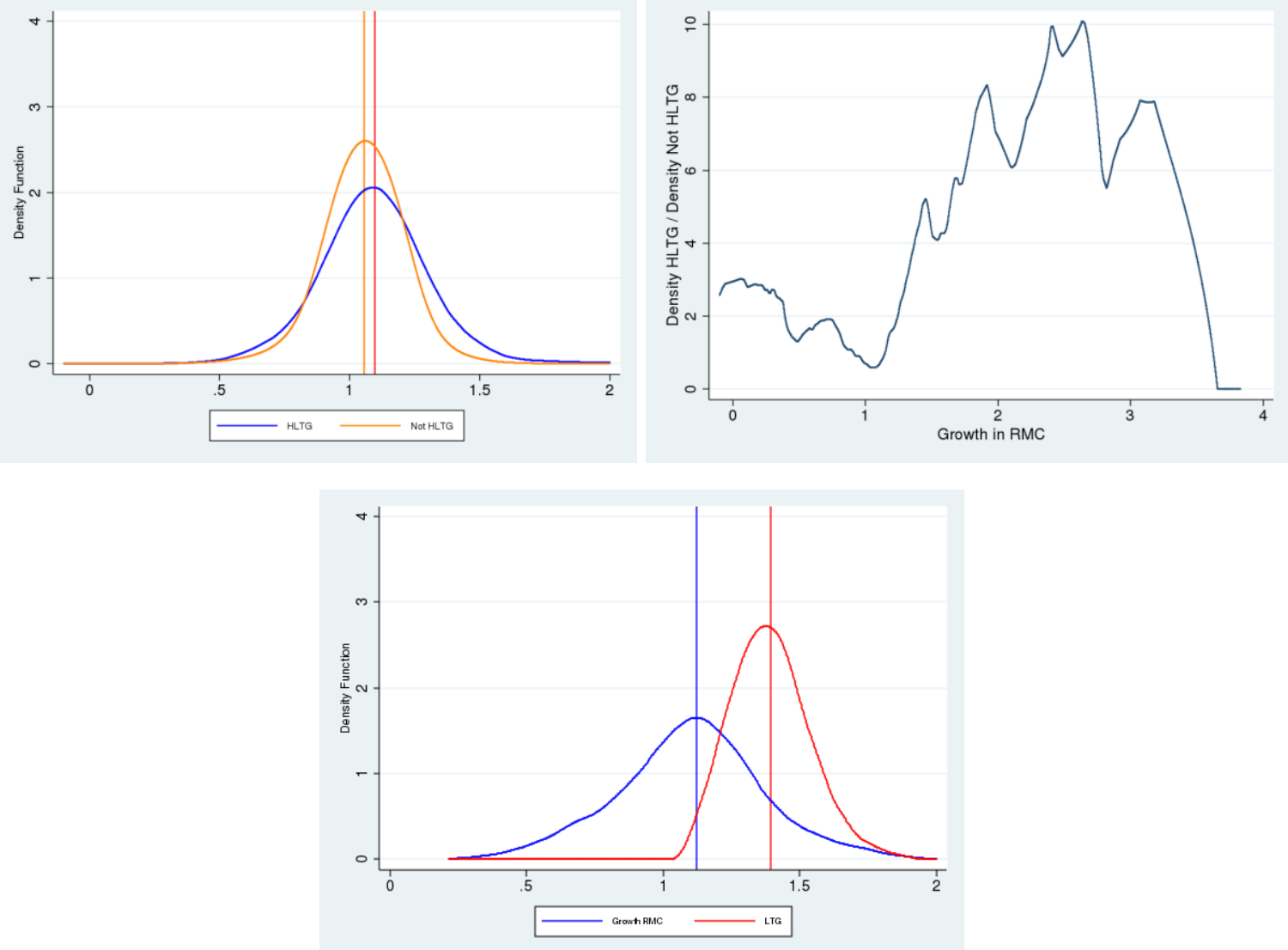

Figure C.3. Long term annualized growth of revenues minus cost of goods sold (RMC) for HLGT portfolio (top left panel). Representativeness of RMC growth for HLTG stocks (top right panel). Growth in RMC and LTG forecasts for HLTG (bottom panel). 


\section{Coibion and Gorodnichenko Analysis}

\section{D.1 Overreaction to News vs Adaptive Expectations}

Adaptive expectations (Equation 11) predict no over-reaction to news after the persistence of the earnings process is accounted for. From (11), the forecast error on an AR(1) process with persistence $\rho$ is $x_{t+1}-x_{t+1}^{a}=(\rho-1) x_{t}+\left(\frac{1-\mu}{\mu}\right)\left(x_{t+1}^{a}-x_{t}^{a}\right)$. Controlling for $x_{t}$ fully accounts for mechanical over-reaction in processes with low persistence. The adaptive forecast revision $\left(x_{t+1}^{a}-x_{t}^{a}\right)$ should positively predict forecast errors as in the under-reaction models. This prediction is not shared by our model because diagnostic expectations over-react to news regardless of the persistence of the data generating process. Table C.1 reports the results. The coefficients on forecast revision become larger than those estimated in Table 2, but they remain mostly negative and statistically significant.

\section{Table D.1: Forecast Errors}

Each entry in the table corresponds to the estimated coefficient of the forecast errors $\left(\mathrm{eps}_{\mathrm{t}+\mathrm{n}} / \mathrm{eps}_{\mathrm{t}}\right)^{1 / \mathrm{n}_{-}} \mathrm{LTG}_{\mathrm{t}}$ for $n=3,4$, and 5 on the variables listed in the first column of the table as well as $(\log )$ eps $_{t}$ and year fixedeffects (not shown).

\begin{tabular}{lccc} 
& \multicolumn{3}{c}{ Dependent Variable } \\
& & & \\
& $\left(\mathrm{eps}_{\mathrm{t}+3} / \mathrm{eps}_{\mathrm{t}}\right)^{1 / 3}-\mathrm{LTG}_{\mathrm{t}}$ & $\left(\mathrm{eps}_{\mathrm{t}+4} / \mathrm{eps}_{\mathrm{t}}\right)^{1 / 4}-\mathrm{LTG}_{\mathrm{t}}$ & $\left(\mathrm{eps}_{\mathrm{t}+5} / \mathrm{eps}_{\mathrm{t}}\right)^{1 / 5}-\mathrm{LTG}_{\mathrm{t}}$ \\
\cline { 2 - 2 } $\mathrm{LTG}_{\mathrm{t}-1}$ & 0.0332 & -0.0733 & $-0.1372^{\mathrm{b}}$ \\
& $(0.0725)$ & $(0.0660)$ & $(0.0589)$ \\
$\mathrm{LTG}_{\mathrm{t}}-\mathrm{LTG}_{\mathrm{t}-2}$ & & & $-0.1842^{\mathrm{a}}$ \\
& -0.0875 & $-0.1435^{\mathrm{b}}$ & $(0.0545)$ \\
& $(0.0641)$ & $(0.0691)$ & \\
$\mathrm{LTG}_{\mathrm{t}}-\mathrm{LTG}_{\mathrm{t}-3}$ & & & $-0.1701^{\mathrm{a}}$ \\
& -0.0956 & $-0.1184^{\mathrm{c}}$ & $(0.0517)$ \\
\hline
\end{tabular}




\section{D.2 Underreaction for forecasts at short time horizons}

Table D. 2 tests the predictability of forecast errors in the forecast of earnings levels, as opposed to the predictability of errors in LTG forecasts analysed in Table 2. The results suggest underreaction for forecasts at short time horizons (i.e. one year ahead), compatible with Bouchaud et al. (2016). As in Table 2, as the forecasting horizon increases to three years ahead, the coefficient becomes less positive and even negative in some specifications.

\section{Table D.2: EPS Forecast Errors at short time horizons}

Each entry in the table corresponds to the estimated coefficient of the forecast errors for $t+1, t+3$, and $\mathrm{t}+5$ on the variables listed in the first column of the table as well as year fixed-effects (not shown). All forecast errors are scaled by lagged sales per share.

\begin{tabular}{|c|c|c|c|}
\hline & $\left(e p s_{t+1}-E_{t} e p s_{t+1}\right) / s p s t_{t-1}$ & $\left(e p s_{t+3}-E_{t} e p s_{t+3}\right) / s p s_{t-1}$ & $\left(e^{e p s_{t+5}-E_{t} e p s_{t+2}} *\left(1+L T G_{t}\right)^{3}\right) / s p s_{t-1}$ \\
\hline \multirow[t]{2}{*}{$\mathrm{LTG}_{\mathrm{t}}-\mathrm{LTG}_{\mathrm{t}-1}$} & 0.0839 & $-0.3226^{b}$ & $-0.5918^{a}$ \\
\hline & $(0.0554)$ & $(0.1312)$ & $(0.1435)$ \\
\hline \multirow[t]{2}{*}{$\mathrm{LTG}_{\mathrm{t}}-\mathrm{LTG}_{\mathrm{t}-2}$} & $0.1629^{a}$ & $0.1262^{c}$ & -0.0227 \\
\hline & $(0.0275)$ & $(0.0677)$ & $(0.0772)$ \\
\hline \multirow[t]{2}{*}{$\mathrm{LTG}_{\mathrm{t}}-\mathrm{LTG}_{\mathrm{t}-3}$} & $0.0825^{a}$ & -0.0664 & $-0.2145^{b}$ \\
\hline & $(0.0195)$ & $(0.0532)$ & $(0.0919)$ \\
\hline
\end{tabular}

\section{D.3 Overreaction and predictable returns}

We next try to tie over-reaction to news to the return spread between HLTG and LLTG portfolios. We estimate Equation (10) by pooling firms at the industry level, using the Fama and French classification. To capture industry and firm specific factors, we allow for industry $\times$ year fixed effects. This yields an industry level estimate $\gamma_{s}$, where $s$ indexes the industry, which we can correlate with the industry level LLTG-HLTG spread. These results should be taken with caution, due to the small number of industries. 
In Figure D.1, we compare the post formation return spread across different terciles of the distribution of industry $\gamma_{s}$. Consistent with our prediction, the extra return obtained by betting against HLTG firms is highest in sectors that feature most over-reaction, namely those in the bottom tercile of $\gamma_{s}$. The return differential is sizable, though given the small sample size it is not statistically significant. Thus, the pattern of LLTG-HLTG return spreads across industries is consistent with a link from overreaction to news to overvaluation of HLTG stocks and thus to abnormally low returns of the HLTG portfolio.

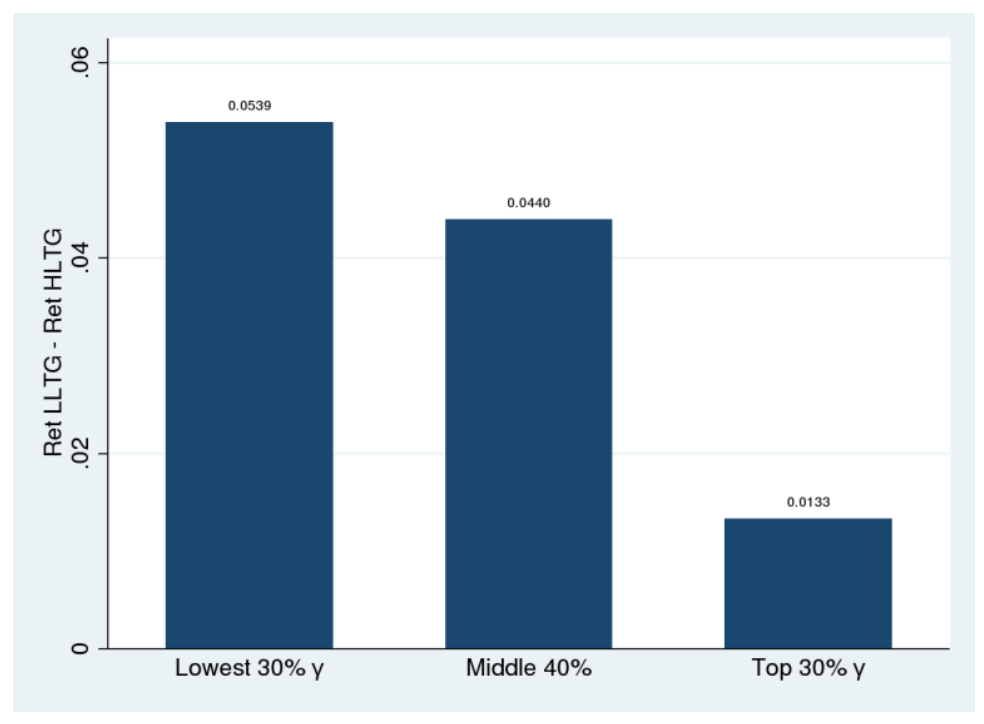

Figure D.1. Overreaction and return spread across industries. For each of the 48 Fama-French industries, we estimate the regression: $\left(E P S_{t+4} / E P S_{t}\right)^{1 / 4}-\left(1+L T G_{t}\right)=\alpha_{i}+\mu_{i, t}+\gamma_{i}\left(L T G_{t}-L T G_{t-3}\right)+\varepsilon_{i, t}$ where $\mu_{\mathrm{t}}$ are year fixed effects, EPS is earnings per share, and LTG is the forecast long-term growth in earnings. We rank industries according to $\gamma_{i s}$ and form the following three groups: (1) 14 industries with the lowest $\gamma_{i S}$, (2) 24 industries with intermediate values of $\gamma_{i s}$, and (3) 14 industries with the highest $\gamma_{i s}$. Finally, for each year and each group, we compute the difference in return for the LLTG (i.e. bottom 30\% of LTG) and HLTG (i.e. highest $30 \%$ of LTG) portfolios. The graph shows the arithmetic mean of the LLTG-HLTG spread for grouping industries based on $\gamma_{i S}$ from the regression. 


\section{E. Calibration}

In this section, we extend our analysis of the calibration with two numerical exercises. The first tests the robustness of our estimate $\theta=1.2$. To do so, we evaluate the matching between the predicted return spread and the empirical return spread for $\theta \in[0,2.4]$ (keeping the other parameters constant). Figure D.1 below shows that the match is indeed optimized for $\theta=1.2$ and that it drops fast as $\theta$ departs from this value.

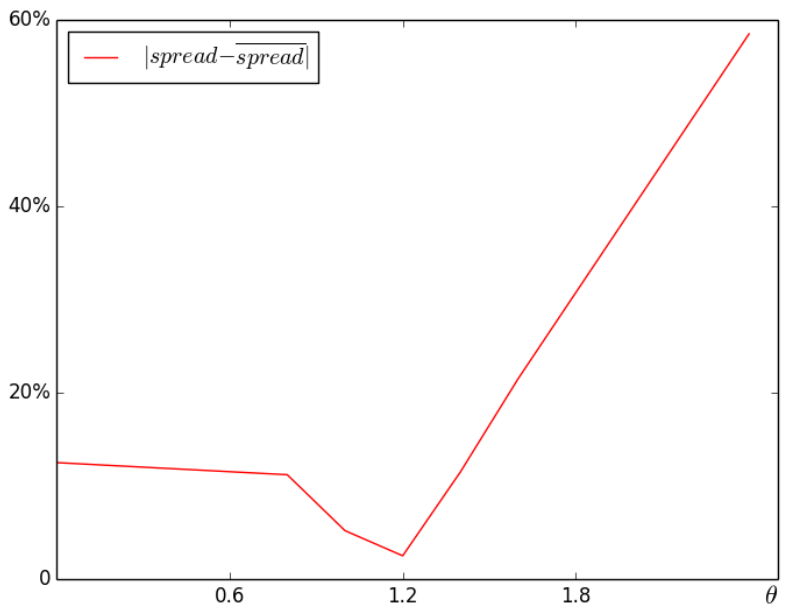

Figure E.1. For $\left(a, b, \sigma_{\eta}, \sigma_{\epsilon}\right)=(0.9,0.33,0.15,0.17)$, as given by our calibration, and each $\theta \in[0,2.4]$ we compute the LLTG-HLTG return spread $\left(\operatorname{spread}_{\theta}\right)$. The figure plots the absolute distance $\mid$ spread $_{\theta}-\overline{\text { spread }} \mid$ where $\overline{\text { spread }}=12.5 \%$ is the empirically measured return spread.

The second exercise is to confirm numerically the pattern of Figure 9, namely that high LTG forecasts are on average revised downwards, even conditional on positive news, that is, earnings that exceeded (distorted) expectations. Figure D.2 below plots the equivalent of Figure 10 for our calibration. 


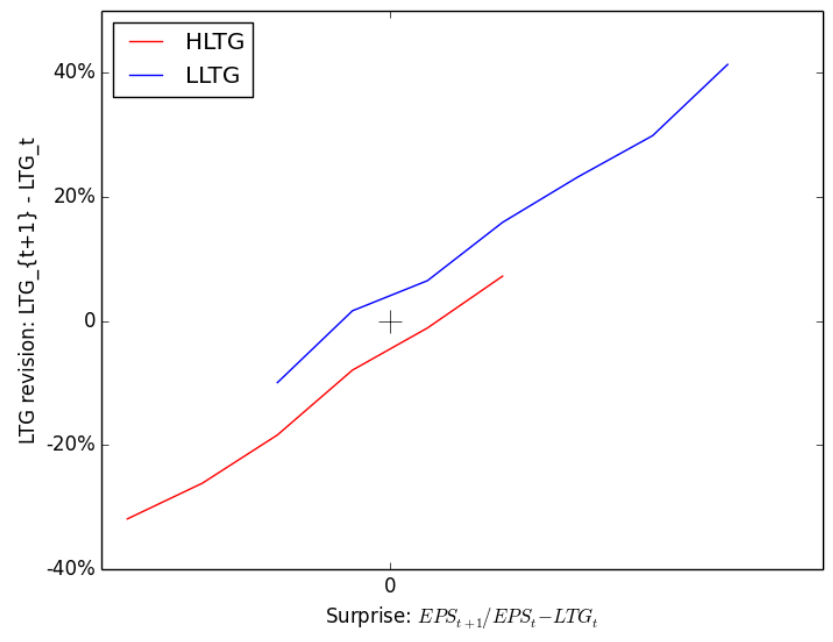

Figure E.2. For each period $t$ and each firm in the $H L T G_{t}$ and $L L T G_{t}$ portfolios, we compute the surprise $\ln E P S_{t+1}-\ln E P S_{t}-L T G_{t}$. Pooling the data for all $t$ and both portfolios, we rank the surprises in deciles and plot the average revision of $L T G$ for each portfolio in each decile.

In the model, news and forecast revisions are positively correlated. Crucially, however, they may go in opposite directions. For a range of positive surprises - namely, realized growth above the diagnostic forecasts - forecasts about HLTG firms are still revised downwards. Naturally, for sufficiently large positive surprises (larger than $1.5 \sigma_{\epsilon}$, in our calibration) forecast revisions are positive. The converse holds for LLTG. 